\title{
Texture formation in orthorhombic alpha-uranium under simple compression and rolling to high strains
}

\author{
Miroslav Zecevic ${ }^{\mathrm{a}}$, Marko Knezevic ${ }^{\mathrm{a},{ }^{*}}$, Irene J. Beyerlein $^{\mathrm{b}}$, Rodney. J. McCabe ${ }^{\mathrm{c}}$ \\ ${ }^{a}$ Department of Mechanical Engineering, University of New Hampshire, Durham, NH 03824, USA \\ ${ }^{\mathrm{b}}$ Theoretical Division, Los Alamos National Laboratory, Los Alamos, NM 87545, USA \\ ${ }^{c}$ Materials Science and Technology Division, Los Alamos National Laboratory, Los Alamos, NM 87545, USA
}

\begin{abstract}
We study the mechanical response and texture evolution of alpha-uranium during simple compression and rolling at $573 \mathrm{~K}$. In order to determine the underlying mechanisms governing plasticity and texture formation, we perform detailed characterizations using electron backscattered diffraction and constitutive modeling using a dislocation-density based hardening law within a visco-plastic self-consistent homogenization. We show that the model achieves good agreement with experimental measurements in terms of texture and stress-strain response. From detailed comparison of experimental and modeling results, we infer that in both throughthickness compression (TTC) and rolling at 573K, the active slip modes are floor slip (001)[100] and chimney slip $1 / 2\{110\}\langle 1 \overline{1} 0\rangle$ with slightly different ratios. However, $\{130\}\langle 3 \overline{1} 0\rangle$ twinning is not active in TTC compression but profuse during rolling. Further analysis indicates that during rolling, floor slip (001)[100] results in the formation of two pronounced (001) texture peaks tilted $10-15^{\circ}$ away from the normal toward the rolling direction.
\end{abstract}

Keywords: Uranium; Texture; Rolling; Dislocations; Twinning

${ }^{*}$ Corresponding author at: Department of Mechanical Engineering, University of New Hampshire, 33 Academic Way, Kingsbury Hall, W119, Durham, New Hampshire 03824, USA. Tel.: +1 603862 5179; fax: +1 603862 1865; E-mail address: marko.knezevic@unh.edu 


\section{Introduction}

Polycrystalline uranium (U) is a metal that is desired for its nuclear properties and high density. The manufacturing of $U$ components involves complex sequences of thermo-mechanical processes, such as forging, rolling, swaging, extrusion, and recrystallization. As the metal undergoes these processes, its microstructure, including grain sizes and shapes, crystal orientations, and defect densities, continuously evolves. The final microstructure, which significantly depends on the processing path taken, determines its mechanical properties and performance in service. For understanding the structural properties of $U$, it is essential to understand its microstructural evolution as a function of processing path and the responsible underlying deformation mechanisms leading to microstructure formation. Such understanding would facilitate designing the processing path for prescribed material properties.

Microstructural evolution is linked to the predominant deformation mechanisms, which for $U$ are much more diverse and complex than cubic materials, such as $\mathrm{Al}, \mathrm{Ni}$, and steels. Pure U, over the pressures and temperatures tested here, has an orthorhombic crystal structure and is referred to as $\alpha$-uranium $(\alpha-U)$ with lattice parameters $a=0.2852 \mathrm{~nm}, \mathrm{~b}=0.5865 \mathrm{~nm}$, and $\mathrm{c}=0.4945 \mathrm{~nm}$ [1]. To deform plastically, $\alpha-U$ exhibits multiple slip and twinning modes. The geometry of the predominant slip/twin systems has been depicted in [2, 3]. Like most metals, possessing a lowsymmetry crystal structure [4-6], activation barriers of these modes are not equivalent. It is known that the easiest slip mode is (010)[100] [7, 8] possessing only one slip system. The (001)[100] slip mode operates at room and becomes the primary slip mode at elevated temperatures $[7,8]$, and is also comprised of only a single slip system. The next easiest slip mode is the $1 / 2\{110\}\langle 1 \overline{1} 0\rangle$ slip mode, which has two slip systems. Finally, the $1 / 2\{1 \overline{1} 2\}\langle 021\rangle$ slip mode is necessary to accommodate plastic strain in the [001] direction and, thus, to accommodate an arbitrary plastic strain. However, its activation requires a larger driving force than the other slip modes. The most commonly observed twinning modes are $\{130\}\langle 3 \overline{1} 0\rangle$, $\{172\}\langle 3 \overline{1} 2\rangle$ and $\{112\}\langle 3 \overline{7} 2\rangle[1,8,9]$, with the former being the easiest to activate. The twin shear $S$ of the $\{130\}\langle 3 \overline{1} 0\rangle$ twin is 0.299 and it reorients the lattice by $69.3^{\circ}$ about [001]. For the $\{172\}\langle 3 \overline{1} 2\rangle$ twin, $S=0.227$, and it reorients the lattice by $92.6^{\circ}$ about $\langle\overline{10} 70\rangle$.

Making the link between these mechanisms and the deformation response of $\alpha-U$ is usually accomplished by crystal plasticity based constitutive models [1, 2, 8-15]. It is generally found that the multiple slip and twinning modes are active within individual crystals of $\alpha$-U and such complex combinations are needed to explain its strong plastic anisotropy in macroscopic behavior [2, 16, 17]. Further, the slip modes differ in their sensitivity to temperature and strain rate leading to interesting and substantial changes in the constitutive response as temperature or strain rate changes [18]. This sensitivity can lead to significant thermal residual stresses upon cooling from the processing temperature to room temperature [14, 19].

It has been shown that the deformation modes have very different activation stresses, and the active modes are significantly affected by the initial texture [16]. Such studies on texture effects have involved mechanical tests involving monotonic deformation. In forming operations like rolling, the deformation state is not as homogeneous as in uniaxial tests. In particular, the deformation state near the surface where the rolls contact the sheet is usually different than in the center [20]. Consequently, many of the models for texture evolution applied in prior works on monotonic deformation would not be well suited to treat texture development during rolling. Furthermore, because $\alpha-U$ is a relatively strong metal that is hard to work at ambient conditions, 
rolling is carried out at elevated temperatures. Up to now, a fundamental analysis of texture development during large strain, elevated temperature rolling of $\alpha-U$ has not been carried out.

In this work, we study texture formation in $\alpha-\mathrm{U}$ during simple compression and rolling using a combination of experiments and multi-scale crystal plasticity based modeling. We explore the effects of initial texture by fabricating samples with approximately the same grain size but different initial textures on the rate of texture formation and concomitant material response in compression. The fabricated material is subsequently rolled at $573 \mathrm{~K}$ to various strain levels and gradients in texture through the thickness of the sheet are characterized. The chief underlying deformation mechanisms responsible for the evolution of texture in $\alpha-\mathrm{U}$ during compression and rolling to large reductions are identified to be as follows: (001)[100] and $1 / 2\{110\}\langle 1 \overline{1} 0\rangle$ slip models are active during both through-thickness compression (TTC) and rolling at 573K with slightly different ratios, $\{130\}\langle 3 \overline{1} 0\rangle$ twinning is not active in TTC compression but profuse during rolling. Analysis shows that during rolling, (001)[100] results in the formation of two pronounced (001) texture peaks tilted $10-15^{\circ}$ away from the normal toward the rolling direction. We demonstrate that the multi-scale coupled with finite elements can capture the development of texture gradients in $\alpha-\mathrm{U}$, a metal with a highly anisotropic plastic response.

\section{Multiscale modeling approach}

Linking the activation of slip and twinning within individual crystals to the imposed deformation conditions, such as rolling, requires linking deformation models from the subgrain scale to the sample scale [21-37]. To this end, we employ a multi-scale model to relate heterogeneous texture evolution at the scale of the sheet material to the deformation mechanisms occurring within a crystal. At the largest scale, we model the deformation of the sample as it is rolled using finite elements (FE). For the constitutive response at each integration point, we use a polycrystal plasticity model called the visco-plastic self-consistent (VPSC) scheme [38]. VPSC relates the deformation of the individual constituent crystals to that of the polycrystal. At the grain scale, we use crystal plasticity theory to relate the deformation of the individual crystal to its active slip and twinning modes. In this work, the shearing action provided by slip and twinning is modeled using a rate-sensitive flow rule [39-42]. To model lattice reorientation due to deformation twinning at the grain scale, we employ the composite grain model (TW) [43-45]. At the subgrain scale, the threshold stresses for activating slip and twinning on individual systems are calculated. For slip, the threshold stresses are governed by the evolution of stored dislocation density (DD) on the slip system according to thermally activated rate laws and hence are explicit functions of temperature and strain rate [18, 46-54]. For twinning, the threshold stresses to first form twins also evolve with strain [46]. The stored dislocation density within the same grain, however, may indirectly affect the expansion of twin lamellae.

\subsection{Sample scale: linking finite element method and VPSC}

In the present model, each FE integration point is a polycrystal whose stress-strain response and microstructure evolution, such as crystallographic and morphologic texture and hardening evolution, are provided by the VPSC model. This model has been implemented as User Material Subroutine (UMAT) for the FE software Abaqus [55-58].

Inside the UMAT, the strain increment from Abaqus, $\Delta \boldsymbol{\varepsilon}^{F E}$, stress from the previous increment, $\boldsymbol{\sigma}^{t}$, and a set of state variables are provided as the input at each material point at each 
trial time increment, $t+\Delta t$. The strain increment results from the applied loading and the associated nodal displacement increments. As the output, the updated stress, $\boldsymbol{\sigma}^{t+\Delta t}$, and tangent stiffness matrix (Jacobian), $\mathbf{C}^{t g}=\partial \Delta \boldsymbol{\sigma} / \partial \Delta \boldsymbol{\varepsilon}$, are calculated and returned. Once the stress field corresponding to the currently applied displacement field is calculated, equilibrium is checked. If not satisfied, increments in displacement are iteratively modified by the Jacobian until equilibrium is achieved. Finally, the same set of state variables are updated and returned.

In our formulation, at each polycrystalline integration point only the corotational increment in stress, $\Delta \boldsymbol{\sigma}$, [59] needs to be calculated since the rigid body rotation is performed within ABAQUS on stress at start of the time increment as $\boldsymbol{\sigma}^{t}=\Delta \mathbf{R} \boldsymbol{\sigma}^{\text {t,unrotated }} \Delta \mathbf{R}^{\mathrm{T}}$ [60-62]. Here, the stress-strain relationship at an integration point follows Hooke's law:

$$
\boldsymbol{\sigma}^{t+\Delta t}=\boldsymbol{\sigma}^{t}+\Delta \boldsymbol{\sigma}=\boldsymbol{\sigma}^{t}+\mathbf{C}:\left(\Delta \boldsymbol{\varepsilon}-\Delta \boldsymbol{\varepsilon}_{v p}\right)
$$

where $\mathbf{C}$ is the elastic stiffness tensor, $\Delta \boldsymbol{\sigma}$ is the corotational increment in the Cauchy stress (calculated in the corotational material frame and then transformed to the fixed global frame), and $\Delta \boldsymbol{\varepsilon}_{v p}$ is the increment in visco-plastic strain calculated by the VPSC model. The elastic stiffness, $\mathbf{C}$, is calculated using the elastic self-consistent (ELSC) code where the textural changes are also accounted for. The single-crystal elastic constants used for $\alpha-U$ are [11, 63]: $\mathrm{C}_{11}=214.8 \mathrm{GPa}, \mathrm{C}_{22}=198.6 \mathrm{GPa}, \mathrm{C}_{33}=267.1 \mathrm{GPa}, \mathrm{C}_{44}=124.4 \mathrm{GPa}, \mathrm{C}_{55}=73.4 \mathrm{GPa}$, $\mathrm{C}_{66}=74.3 \mathrm{GPa}, \mathrm{C}_{12}=46.5 \mathrm{GPa}, \mathrm{C}_{13}=21.8 \mathrm{GPa}$ and $\mathrm{C}_{23}=107.6 \mathrm{GPa}$.

The procedure for finding the stress increment is described in $[55,64]$ and is briefly summarized here. Rewriting Eq. (1), we obtain:

$$
\Delta \boldsymbol{\varepsilon}=\mathbf{C}^{-1}: \Delta \boldsymbol{\sigma}+\Delta t \dot{\boldsymbol{\varepsilon}}_{v p}^{(p x)}\left(\boldsymbol{\sigma}^{t}+\Delta \boldsymbol{\sigma}\right)
$$

For a given trial strain increment, $\Delta \boldsymbol{\varepsilon}^{F E}$, we define the residual $\mathbf{X}=\mathbf{X}(\Delta \boldsymbol{\sigma})$ at each integration point as a nonlinear function of the stress increment $\Delta \sigma$ :

$$
\mathbf{X}(\Delta \boldsymbol{\sigma})=\Delta \boldsymbol{\varepsilon}-\Delta \boldsymbol{\varepsilon}^{F E}=\mathbf{C}^{-1}: \Delta \boldsymbol{\sigma}+\Delta t \dot{\boldsymbol{\varepsilon}}_{v p}^{(p x)}\left(\boldsymbol{\sigma}^{t}+\Delta \boldsymbol{\sigma}\right)-\Delta \boldsymbol{\varepsilon}^{F E}
$$

The condition $\mathbf{X}(\Delta \boldsymbol{\sigma})=\mathbf{0}$ (i.e. $\Delta \boldsymbol{\varepsilon}=\Delta \boldsymbol{\varepsilon}^{F E}$ ) is enforced using a Newton-Raphson (NR) scheme to solve the nonlinear system of equations. The corresponding Jacobian $\mathbf{J}_{N R}$ is given by:

$$
\frac{\partial \mathbf{X}(\Delta \boldsymbol{\sigma})}{\partial \Delta \boldsymbol{\sigma}}=\mathbf{J}_{N R}=\mathbf{C}^{-1}+\Delta t \frac{\partial \dot{\boldsymbol{\varepsilon}}_{v p}^{(p x)}}{\partial \Delta \boldsymbol{\sigma}}\left(\boldsymbol{\sigma}^{t}+\Delta \boldsymbol{\sigma}\right)=\mathbf{C}^{-1}+\Delta t \mathbf{M}^{(p x)}\left(\boldsymbol{\sigma}^{t}+\Delta \boldsymbol{\sigma}\right)
$$

Hence, given a guess $\Delta \boldsymbol{\sigma}^{k-1}$ for the stress increment, the new guess is obtained as:

$$
\Delta \boldsymbol{\sigma}^{k}=\Delta \boldsymbol{\sigma}^{k-1}-\mathbf{J}_{N R}^{-1}\left(\Delta \boldsymbol{\sigma}^{k-1}\right): \mathbf{X}\left(\Delta \boldsymbol{\sigma}^{k-1}\right)
$$

From Eq. (3), it is easy to recognize that Eq (4) also provides a closed expression for the FE Jacobian as a function of the visco-plastic tangent moduli (described next), the elastic stiffness of the aggregate, and the FE time increment:

$$
\mathbf{C}^{t g}=\frac{\partial \Delta \boldsymbol{\sigma}}{\partial \Delta \varepsilon}=\left(\frac{\partial \Delta \varepsilon}{\partial \Delta \sigma}\right)^{-1}=\left(\frac{\partial \mathbf{X}(\Delta \boldsymbol{\sigma})}{\partial \Delta \boldsymbol{\sigma}}\right)^{-1}=\mathbf{J}_{N R}^{-1}
$$

The internal state variables such as twins, crystallographic orientations, axis of the ellipsoids representing grains, orientation of these ellipsoids and slip/twin resistances are updated upon reaching a solution at a given strain increment.

\subsection{Polycrystal scale: linking visco-plastic self-consistent model and TW and DD}

In VPSC, a polycrystal is represented by a number of grains, each with an assigned crystallographic orientation, shape and volume fraction. To calculate its interaction with the other grains in the polycrystal, each grain is treated as a visco-plastic inclusion embedded in a homogeneous effective medium (HEM). This elementary inclusion problem is solved using a 
Green's function approach [38]. Each inclusion (or representative grain) may deform via a combination of crystallographic slip and twinning. The slip or twin shearing rate, $\dot{\gamma}^{s}$, on a system, $s$, is related to the resolved shear stress, $\tau^{s}$, on that system by:

$$
\dot{\gamma}^{s}=\dot{\gamma}_{0}\left|\frac{\boldsymbol{\sigma}^{\prime} \cdot \mathbf{m}^{s}}{\tau_{c}^{s}}\right|^{\frac{1}{m}} \operatorname{sign}\left(\boldsymbol{\sigma}^{\prime} \cdot \mathbf{m}^{s}\right) .
$$

where $\dot{\gamma_{0}}$ is the reference slip rate, $m$ is the rate sensitivity parameter, $\mathbf{m}^{s}$ is the Schmid tensor for slip or twinning system $s$ and $\tau_{c}^{s}$ is the threshold stress on the system. Here we set $\dot{\gamma}_{0}$ to be the macroscopic strain rate and $1 / m$ to be 10 . The Cauchy stress deviator $\boldsymbol{\sigma}^{\prime}$ and the viscoplastic strain-rate $\dot{\boldsymbol{\varepsilon}}_{v p}$ at the single-crystal level are related by:

$$
\dot{\boldsymbol{\varepsilon}}_{v p}(\mathbf{x})=\sum_{s=1}^{N_{s}} \mathbf{m}^{s}(\mathbf{x}) \dot{\gamma}^{s}(\mathbf{x})
$$

where $N_{S}$ is the total number of slip and twin systems. As in the usual VPSC model, for every representative grain $\mathrm{r}$, the local nonlinear relation in Eq. 8 is linearized, to give:

$$
\dot{\boldsymbol{\varepsilon}}_{v p}^{(r)}=\mathbf{M}^{(r)}: \boldsymbol{\sigma}^{\prime(r)}+\dot{\boldsymbol{\varepsilon}}^{o(r)}
$$

where $\mathbf{M}^{(r)}$ and $\dot{\boldsymbol{\varepsilon}}^{o(r)}$ are, respectively, the linearized viscoplastic compliance and backextrapolated strain-rate of grain $r$. The behavior of the effective medium (polycrystal, $p x$ ) also takes on a similar linear relationship:

$$
\dot{\boldsymbol{\varepsilon}}_{v p}^{(p x)}=\mathbf{M}^{(p x)}: \boldsymbol{\sigma}^{(p x)}+\dot{\boldsymbol{\varepsilon}}^{o(p x)}
$$

where $\dot{\boldsymbol{\varepsilon}}_{v p}^{(p x)}$ is the effective (polycrystal) deviatoric strain-rate tensor, and $\boldsymbol{\sigma}^{(p x)}$ the effective stress tensor, $\mathbf{M}^{(p x)}$ is the tangent viscoplastic compliance, and $\dot{\boldsymbol{\varepsilon}}^{(p x)}$ the back-extrapolated strain-rate of the HEM. The VPSC method then aims to calculate the uniform stress and strain for every ellipsoidal grain with moduli $\mathbf{M}^{(r)}$ and $\dot{\boldsymbol{\varepsilon}}^{o(r)}$ embedded in an infinite medium of moduli $\mathbf{M}^{(p x)}$ and $\dot{\boldsymbol{\varepsilon}}^{o(p x)}$.

\subsection{Grain scale: linking subgrain scale kinetics of activation of slip and twinning}

The flow law Eq. (7) introduces threshold stresses for each slip and twinning system. For dislocation slip, the threshold stress $\tau_{c}^{S}$ evolves as dislocations are stored in the crystal. Stored dislocations are represented as statistical densities and in the present model this density follows thermally activated rate laws [65-67]. In the model, $\tau_{c}^{s}$ is the sum of an initial slip resistance $\tau_{0}^{\alpha}$ (which includes the Peierls stress, the Hall-Petch grain boundary barrier effects associated with the initial grain size and the initial content of dislocations) and obstacle stresses $\tau_{f o r}^{s}$ and $\tau_{d e b}^{\alpha}$ related to the spatially random and ordered distribution of stored dislocations, respectively [46, 68]:

$$
\tau_{c}^{s}=\tau_{0}^{\alpha}+\tau_{\text {for }}^{s}+\tau_{\text {deb }}^{\alpha} .
$$

In what follows, each term is described in turn. A model for the initial slip resistance $\tau_{0}^{\alpha}(T)$ is empirical and assumed to vary with temperature exponentially according to:

$$
\tau_{0}^{\alpha}(T)=A \exp \left(-\frac{T-295}{B}\right),
$$

where $\mathrm{A}$ and $\mathrm{B}$ are material constants, and $T$ is the current temperature. A represents the room temperature resistance and $\mathrm{B}$ determines the temperature dependence. When a grain contains twins, the twin boundaries that form can serves as obstacles to slip. For this purpose the term $\tau_{H P}^{\alpha}$ is added to Eq. (11). It represents the additional barrier effect introduced by twin boundaries on 
the dislocation mean free path. It is modeled based on the empirical Hall-Petch-like effect and is defined per slip system, $s$, as

$$
\tau_{H P}^{S}=\left(\frac{f^{p t s}-f^{p t s, 0}}{f^{p t s, m a x}-f^{p t s, 0}}\right)^{0.1} \mu^{\alpha}(\mathrm{T}) H P^{\alpha} \sqrt{\frac{b^{\alpha}}{d_{m f p}^{s}}},
$$

where $b^{\alpha}$ is the magnitude of the Burgers vector, $H P^{\alpha}$ is the Hall-Petch parameter, $\mu^{\alpha}(T)$ is the shear modulus as a function of temperature and constants $f^{p t s, 0}, f^{p t s, \text { max }}$ are taken to be 0.05 and 0.95 . The shear modulus values per slip/twin mode are taken from [18]. The mean free path $d_{m f p}^{s}$ is calculated using $[43,46]$ :

$$
d_{m f p}^{s}=\frac{\left(1-f^{p t s}\right) d_{c}}{\sin (\theta)}
$$

where the angle $\theta$ is defined between the slip plane of $s$ and the twin plane of the predominant twin system (PTS), $d_{c}=\frac{d_{g}}{n^{\text {lamellae }}}$ is the separation between adjacent twin lamellae, $d_{g}$ is the grain size, and $n^{\text {lamellae }}$ is number of lamellae per grain, which is set equal to 2 , a value that represents the average value seen experimentally.

The resistances arising from stored dislocations, $\tau_{\text {for }}^{S}(\dot{\varepsilon}, T)$ and $\tau_{d e b}^{S}(\dot{\varepsilon}, T)$, are directly related to the stored forest $\rho_{\text {for }}^{S}(\dot{\varepsilon}, T)$ and debris $\rho_{\text {deb }}^{S}(\dot{\varepsilon}, T)$ dislocation densities, via Taylor-type laws $[69,70]$ :

$$
\begin{aligned}
& \tau_{\text {for }}^{s}(\dot{\varepsilon}, T)=b^{\alpha} \mu^{\alpha}(T) \sqrt{\chi^{s s^{\prime}} \rho_{\text {for }}^{s^{\prime}}(\dot{\varepsilon}, T)}, \\
& \tau_{d e b}^{\alpha}(\dot{\varepsilon}, T)=k_{d e b} \mu^{\alpha}(T) b^{\alpha} \sqrt{\rho_{d e b}(\dot{\varepsilon}, T)} \log \left(\frac{1}{b^{\alpha} \sqrt{\rho_{d e b}(\dot{\varepsilon}, T)}}\right),
\end{aligned}
$$

where $\chi^{\text {ss' }}$ is a dislocation-interaction matrix. In this work, we assign only the diagonal terms $\left(s=s^{\prime}\right)$ a non-zero value and further use a typical value of 0.81 [69].

The dislocation density, $\rho_{\text {for }}^{S}(\dot{\varepsilon}, T)$, evolves based on a balance between the rate of dislocation storage and the rate of dislocation recovery following the theory on the thermodynamics of slip $[69,71]$ :

$$
\frac{\partial \rho_{\text {for }}^{s}}{\partial \gamma^{s^{\prime}}}=\frac{\partial \rho_{\text {gen, for }}^{s}}{\partial \gamma^{s^{\prime}}}-\frac{\partial \rho_{\text {rem, for }}^{s}}{\partial \gamma^{s^{\prime}}}=k_{1}^{s} \sqrt{\rho_{\text {for }}^{s}}-k_{2}^{s}(\dot{\varepsilon}, \mathrm{T}) \rho_{\text {for }}^{s} .
$$

In Eq. (16), $k_{1}^{s}$ is a rate-insensitive coefficient for dislocation storage by statistical trapping of dislocations, whereas $k_{2}^{s}$ is a rate-sensitive coefficient for dynamic recovery by thermally activated mechanisms. $k_{2}^{s}$ is given by:

$$
\frac{k_{2}^{s}}{k_{1}^{s}}=\frac{1}{\sqrt{\left(\chi^{-1}\right)^{s s^{\prime}} \frac{\tau_{s a t}^{s^{\prime}}}{b^{\alpha^{\prime}} \mu^{\alpha^{\prime}}}}}, \tau_{\text {sat }}^{s}=\frac{D^{\alpha}\left(b^{\alpha}\right)^{3} g^{\alpha} \mu^{\alpha}}{D^{\alpha}\left(b^{\alpha}\right)^{3}-k T \ln \left(\frac{\dot{\varepsilon}}{\dot{\varepsilon}_{0}}\right)}
$$

where $\chi^{\text {ss' }}$ is the interaction matrix defined earlier, and $k, \dot{\varepsilon}_{o}, g^{\alpha}$, and $D^{\alpha}$ are the Boltzmann's constant, a reference strain rate equal to $10^{7} \mathrm{~s}^{-1}$, an effective activation enthalpy, and a dislocation drag stress, respectively. Dynamic recovery is often associated with thermally activated dislocation processes such as dislocation cross-slip and climb. Accompanying such processes is the production of dislocation debris. Accordingly the rate of debris $\rho_{d e b}^{S}(\dot{\varepsilon}, T)$ accumulation is related to the rate of recovery through: 


$$
d \rho_{d e b}=q^{\alpha} b^{\alpha} \sqrt{\rho_{d e b}} \frac{\partial \rho_{r e m, f o r}^{s}}{\partial \gamma^{s}}\left|d \gamma^{s}\right|
$$

where $q^{\alpha}$ is a rate coefficient. It can be viewed as the fraction of recovered dislocations that is stored as debris.

The hardening model described above differs in some aspects from that used previously for U. Here, the dislocation storage term, $k_{2}$, can take into account dislocation interactions of various strength while in the previous model it was a function of only the average dislocation interaction coefficient.

The threshold stresses for activating twinning follow a different model than one described above for slip. For a given twin mode $\beta$ and each of its twin systems $t$, a threshold stress is modeled as a sum of resistances:

$$
\tau_{c}^{\beta}=\tau_{0}^{\beta}+\tau_{\text {slip }}^{\beta}
$$

where $\tau_{0}^{\beta}$ is a temperature-independent friction term and $\tau_{\text {slip }}^{\beta}$ is a latent hardening term that couples slip and twin systems. The latter is given by

$$
\tau_{\text {slip }}^{\beta}=\sum_{s} C^{\alpha \beta} \mu^{\alpha}(\mathrm{T}) b^{\beta} b^{\alpha} \rho_{\text {for }}^{s}
$$

where $C^{\alpha \beta}$ is the latent hardening matrix.

The formation of a twin domain within a grain with a characteristic twin-matrix orientation relationship is modeled by partitioning the grain into a representative twin and matrix inclusion [43]. The crystallographic orientation of the twin domain corresponds to the twinning system with the highest shear-rate among all active twin systems, i.e., the predominant twin system (PTS). The short axes of the matrix and twin ellipsoids are perpendicular to the twin plane. To model twin growth, the ellipsoids representing the twins are allowed to thicken. At the same time, those representing the matrix shrink.

\subsection{Material parameters for the models for threshold stresses}

In all deformation simulations, we make available the following deformation mechanisms: wall slip (010)[100], chimney slip $1 / 2\{110\}\langle 1 \overline{1} 0\rangle$, floor slip (001)[100], roof slip $1 / 2\{021\}\langle 1 \overline{1} 2\rangle,\{130\}\langle 3 \overline{1} 0\rangle$ twinning, and $\{172\}\langle 3 \overline{1} 2\rangle$ twinning. A single set of these parameters is used for all calculations presented here. These parameters are physically based and material dependent and are not adjusted for different extrinsic variables such as initial texture, deformation pathway, strain rate, and temperature.

The parameter set is presented in Tables I and II for slip and twinning, respectively. As expected, we see that among the slip modes, the initial athermal activation stress for (010)[100] is the lowest, (001)[100] the second lowest, $1 / 2\{110\}\langle 1 \overline{1} 0\rangle$ higher, and $1 / 2\{021\}\langle 1 \overline{1} 2\rangle$ the highest. Significantly, this agrees with earlier studies on single crystals [8] as well as prior modeling work [18]. Among the twin modes, we also find that the activation stress for $\{130\}\langle 3 \overline{1} 0\rangle$ is lower than $\{172\}\langle 3 \overline{1} 2\rangle$, which is consistent with earlier single crystal studies [8, 9]. In simulations, we permit de-twinning and secondary (internal) twinning to occur [44, 45, 47], but these mechanisms were not active. 
Table I. Material parameters used in the model for the evolution of slip system resistance.

\begin{tabular}{ccccc}
\hline & Wall slip & Chimney slip & Floor slip & Roof slip \\
& $\alpha=1,(010)[100]$ & $\alpha=2,1 / 2\{110\}\langle 1 \overline{1} 0\rangle$ & $\alpha=3,(001)[100]$ & $\alpha=4,1 / 2\{021\}\langle 1 \overline{1} 2\rangle$ \\
\hline$b^{\alpha}[\mathrm{m}]$ & $2.85 \times 10^{-10}$ & $6.51 \times 10^{-10}$ & $2.85 \times 10^{-10}$ & $11.85 \times 10^{-10}$ \\
\hline$H P^{\alpha=1,2}$ & 50.0 & 50.0 & 300.0 & 50.0 \\
$k_{1}^{\alpha}\left[\mathrm{m}^{-1}\right]$ & $1.2 \times 10^{8}$ & $2.0 \times 10^{7}$ & $1.0 \times 10^{7}$ & $2.2 \times 10^{8}$ \\
$g^{\alpha}$ & 0.01 & 0.01 & 0.01 & 0.01 \\
$D^{\alpha}[\mathrm{MPa}]$ & 446 & 70 & 126 & 126 \\
$q^{\alpha}$ & 18.0 & 4.0 & 0.0 & 80.0 \\
\hline $\mathrm{A}$ & 255 & 655 & 590 & 900 \\
$\mathrm{~B}$ & 380 & 140 & 350 & 940
\end{tabular}

Table II. Material parameters used in the model for the evolution of twin resistance.

\begin{tabular}{ccc}
\hline & $\beta=1,\{130\}\langle 3 \overline{1} 0\rangle$ & $\beta=1,\{172\}\langle 3 \overline{1} 2\rangle$ \\
\hline$b^{\beta}[\mathrm{m}]$ & $10.36 \times 10^{-11}$ & $14.33 \times 10^{-10}$ \\
\hline$\tau_{0}^{\beta}$ & 100.0 & 500.0 \\
$C^{1 \beta}$ & 5500 & 5500 \\
$C^{2 \beta}$ & 5500 & 5500 \\
$C^{3 \beta}$ & 8500 & 8500 \\
$C^{4 \beta}$ & 6800 & 6100
\end{tabular}

\section{Material, processing, and experimental methods}

To study initial texture effects, sample materials with two different starting textures but similar grain sizes were produced $[2,16]$. The materials were initially cast into ingots using a vacuum-induction-melting. The ingots were upset forged and hot rolled at $640 \mathrm{C}$ and then annealed at $480 \mathrm{C}$ for 2 hours. Thereafter, they were warm rolled at $330 \mathrm{C}$ in using two different rolling schedules in order to create two different starting microstructures. In one case, the material was straight rolled (SR) and in the other case it was clocked rolled (CR). In clock rolling, the material is rotated by multiples of $45^{\circ}$ after each pass, whereas in straight rolling, the material orientation is maintained. The final thickness of the sheet in both cases was $8.89 \mathrm{~mm}$. After rolling, the SR and CR materials were annealed at $550 \mathrm{C}$ for two hours.

The textures and grain structure of these two samples were characterized using electronbackscattered diffraction (EBSD). Details on the procedures we used to prepare the samples for EBSD can be found in $[2,18]$. Data were collected from EBSD using a TSL camera attached to a FEI XL30 ESEM at an accelerating voltage of $25 \mathrm{KV}$. For texture measurements, EBSD scans were performed with 5-10 $\mu \mathrm{m}$ step sizes over an area of several square millimeters, while for microstructural analyses, scans were run with a $0.2 \mu \mathrm{m}$ step size over a smaller area of $200 \times 100$ $\mu \mathrm{m}$. 
Figure 1 shows the resulting textures and microstructures. As desired, we see that they differ predominantly in texture and less so in grain size. In both, the grains are equiaxed and twin free with an average grain size of $20 \mu \mathrm{m}$. The SR and CR deformation textures both exhibit a strong (001) component in the through-thickness direction (TT) with the split along in-plane 2-direction (IP2), (010) component in the in-plane 1-direction (IP1) and (100) component in the IP2. However, compared to the SR texture, the CR texture exhibits less in-plane anisotropy, with, for instance, little difference in the intensities of the (010) poles in IP1 and IP2 directions. IP1 is the direction parallel to the final rolling direction (RD) for the CR material and the RD for the SR material, while IP2 is the direction transverse to the RD.

(a)

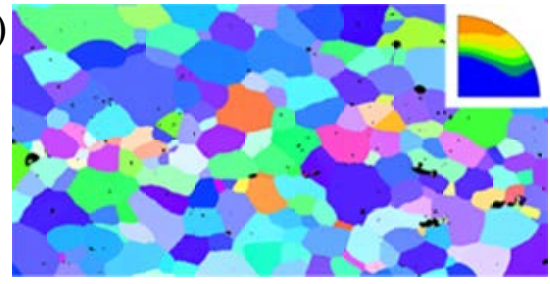

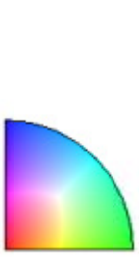

(b)

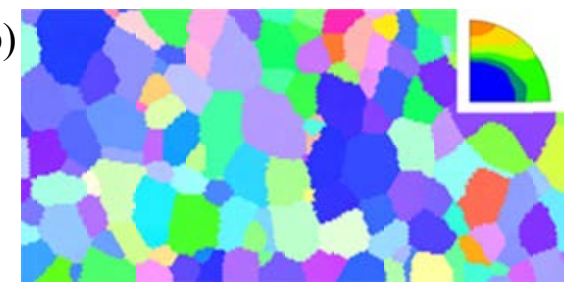
$50 \mu \mathrm{m}$

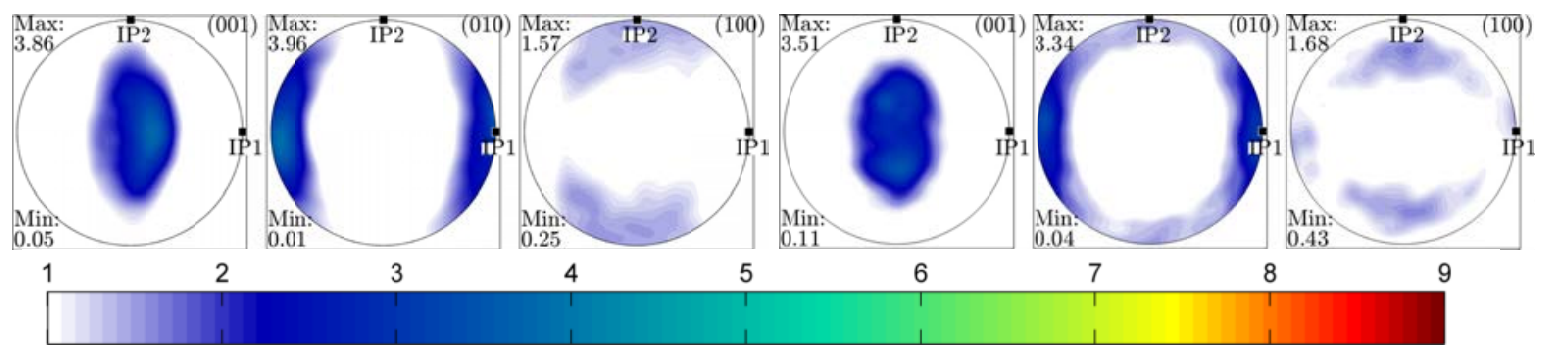

Fig. 1. Orientation maps showing the initial microstructures and pole figures showing the initial textures measured in the as-annealed samples of (a) straight rolled (SR) and (b) clock rolled (CR) uranium. The colors in the orientation maps indicate the orientation of the in-plane 1 direction (IP1) axis with respect to the crystal reference frame. In the corner of each orientation map is the IP1 direction inverse pole figure showing the distribution of grain orientations in the compression direction. All IPF triangles have the crystal reference frame defined as [100] to the right, [010] upward, and [001] in the center.

\section{Results}

\subsection{Simple compression response}

To examine deformation behavior, the straight-rolled (SR) and clocked-rolled (CR) annealed materials were tested in uniaxial compression under different loading conditions. Right-circular cylinder, $6.35 \mathrm{~mm}$ diameter, compression samples were machined in three orientations. Samples were tested in through thickness compression (TTC), in-plane compression in 1 (IPC1, which is the final rolling direction), and in-plane compression in 2 (IPC2, which is the direction perpendicular to the last rolling direction), at temperatures ranging from room temperature to 573 $\mathrm{K}$, and at different quasi-static strain rates $\left(10^{-3} / \mathrm{s}\right.$ to $\left.10^{-1} / \mathrm{s}\right)$. 
To build the starting microstructure of the polycrystal model, we imported the measured initial textures of the SR and CR materials (Fig. 1), which are represented by 10,000 distinct orientations (grains) each with equal volume fraction. Consistent with the initial grain structure seen in Fig. 1, we assigned all of the initial grain shape to be spherical and with diameters $d_{\mathrm{g}}$ of $15 \mu \mathrm{m}$. In the deformation simulations, we impose homogeneous boundary conditions corresponding to uniaxial compression in the prescribed load direction (with respect to the initial texture) and traction-free surfaces in the other two orthogonal directions. To model the effect of the constraint of neighboring grains on grain reorientation, we invoked the co-rotation scheme [72]. In this model, each grain is assigned its child and another, randomly chosen, grain in the polycrystal to serve as its neighbors. Then the average reorientation of grain and its neighbors, weighted by their volume fractions, is calculated and used for grain rotation [73].

Figure 2a compares the measured and calculated stress-strain curves for the SR material. Considering the complexity of the studied material, the calculations agree reasonably well in flow stress and hardening rate for all three directions. The plastic anisotropy is pronounced. The TTC flow stress is higher than the in-plane directions. The stress responses in the two in-plane directions are not equal with the IPC2 being higher than the IPC1.

The plastic anisotropy in flow stress can in part be the result of the initial texture and differences in its evolution in each test. Figure $2 b$ shows the measured deformation textures after each test. We see in Fig. $2 b$ that the texture has changed from the initial state to achieve a strong texture after compression. The final texture after IPC1 is markedly different than that after compression in IPC2 and TTC3. In IPC1, the (001) poles concentrate along the TT direction, the (100) poles along IPC1, which is the direction of loading, and the (010) poles in the IP2 direction. In contrast, for IPC2, the (001) poles are distributed in the TT-IP2 plane. The (100) poles concentrate along the IP2 direction of loading and the $(010)$ poles peak in the IP1 direction. In TTC3, the texture appears weaker than for the in-plane tests. We see the development of two maxima about $15-25^{\circ}$ from the TT direction towards the IP2 direction. There are diffuse and weak (100) peaks along the IP2 direction and diffuse and weak (010) peaks in the IP1 direction. The corresponding model predictions are also provided in these figures and here we see that the agreement is reasonable, considering the complexity of the material. It is noted that the simulated textures evolve too rapidly in (010) and (100) pole figures whereas in the (001) pole figure not quickly enough. 
(b)
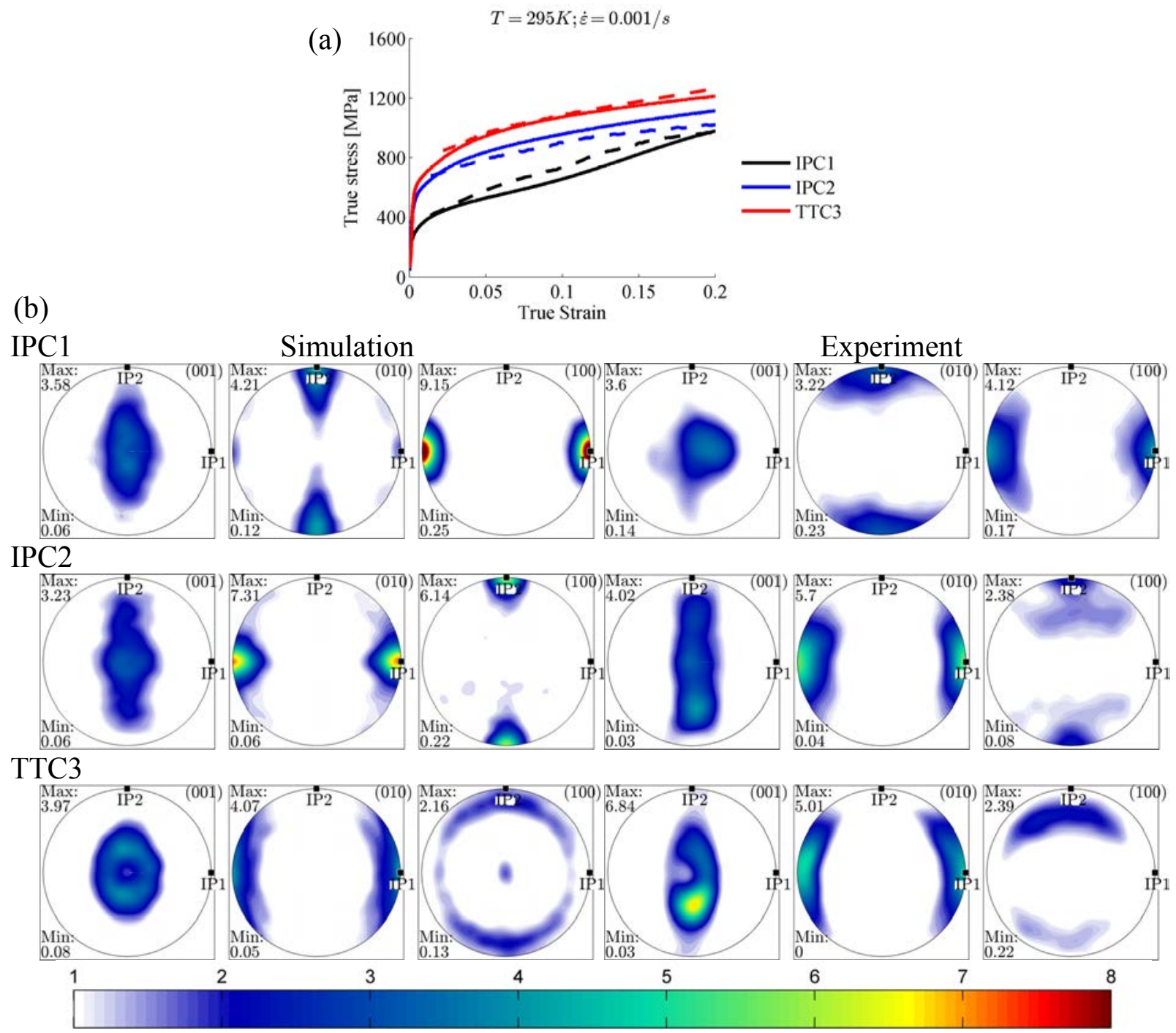

(c)
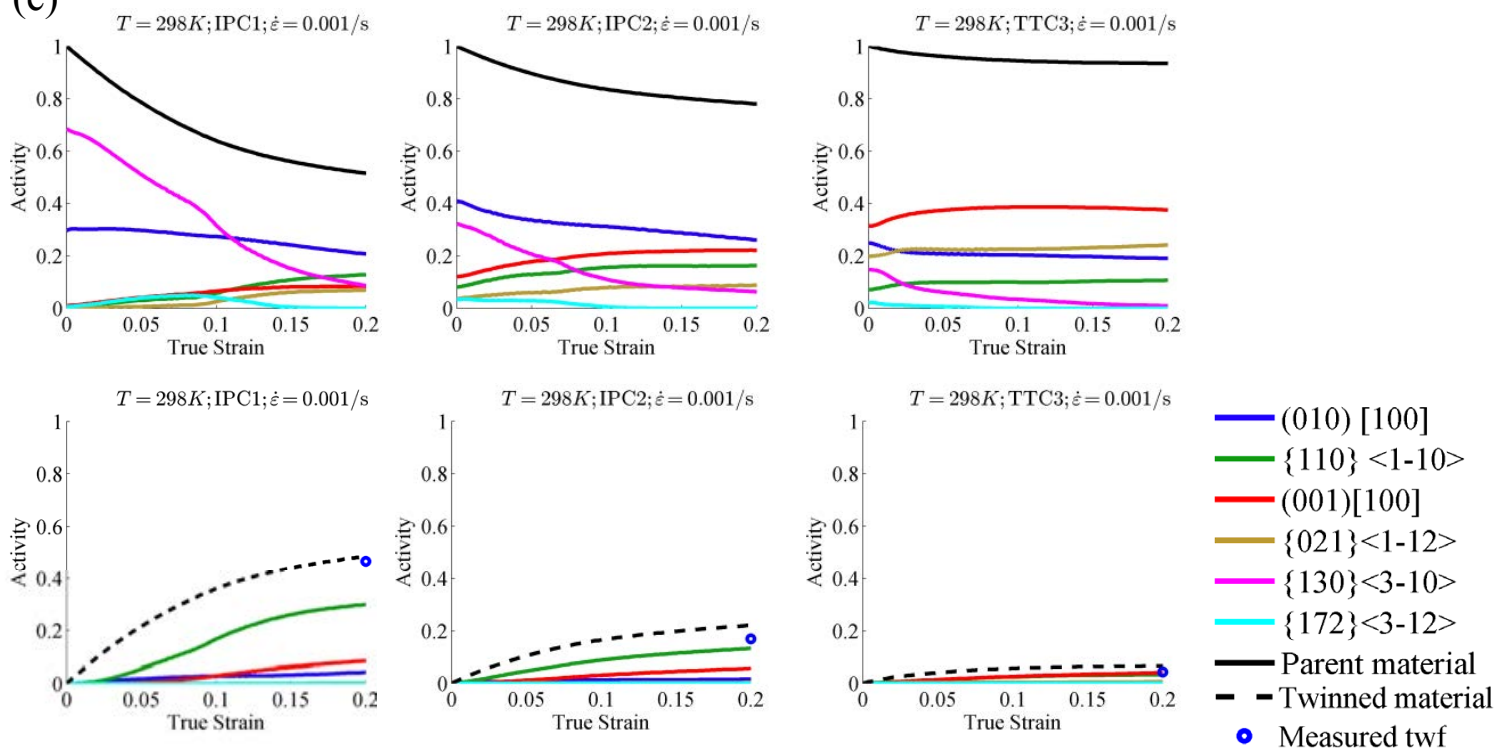
Fig. 2. Comparison of measured vs. simulated (a) stress-strain response and (b) texture evolution at room temperature in three different directions. (c) Relative activities of active deformation modes within parent grains (top row) and within twins (bottom row). Also plotted are the corresponding parent material (top row) and twinned material (bottom row) volume fraction. The initial texture was $S R$.

Development of distinctly different textures indicates that each loading direction activated different deformation mechanisms. One of the outputs of the model is the slip and twin activity responsible for the constitutive response and texture development. For the foregoing compression tests, these predictions are shown in Fig. 2c in the form of the relative activity of each deformation mode as a function of deformation strain. The predicted twin volume fractions show great agreement with the corresponding experimental measurements. Due to the low symmetry crystal structure of $\alpha-\mathrm{U}$, it is not surprising to see that all slip modes and in some cases, both twinning modes, participate in accommodating the deformation. However, the predominant slip mode and the distribution of slip among the active slip modes are strongly dependent on the direction of loading with respect to the initial texture. In the two in-plane compression directions, both twin modes are active and the easiest slip mode, wall slip (010)[100], is the predominant slip mode. However, IPC1 activates much more $\{130\}\langle 3 \overline{1} 0\rangle$ twinning than IPC2, which results in a higher hardening rate than IPC2. More twinning and less activity of the harder floor slip (001)[100] caused IPC1 to have the lower flow stress. Unlike inplane compression, in through-thickness compression, floor slip (001)[100] and roof slip $1 / 2\{021\}\langle 1 \overline{1} 2\rangle$ are the predominant slip modes and wall slip (010)[100] is much less active.

\subsection{Temperature effects}

To examine the effect of elevated temperatures on texture evolution and activation of slip and twinning, compression tests on the CR material were performed at various temperatures between room temperature and $573 \mathrm{~K}$. Figures $3 \mathrm{a}$ and $4 \mathrm{a}$ compare the simulated and measured TTC and IPC2 compression stress-strain curves at room, $373 \mathrm{~K}, 473 \mathrm{~K}$, and $573 \mathrm{~K}$ temperatures. As in the SR case, the agreement between the model and measurement is reasonably good. Moreover, we see pronounced plastic anisotropy between the TTC and IPC2 loading tests. Fig. 3b and 4b show the calculated deformation textures. These model predictions suggest an anisotropic development in the texture accompanies the plastic anisotropy in compression. In the TTC test, the (010) component slightly increases in the IP1 direction as temperature increases. In the IPC2 test, we see that when increasing from $473 \mathrm{~K}$ to $573 \mathrm{~K}$, also slight changes occur, with the appearance of additional components in the (010) and (100) pole figures and a diminished (100) component in the IP2 direction.

Figure $3 \mathrm{c}$ and $4 \mathrm{c}$ present the corresponding slip activities. The two most active slip modes at room temperature for TTC are floor slip (001)[100] and roof slip $1 / 2\{021\}\langle 1 \overline{1} 2\rangle$. Cooperative activity of floor and roof slip causes formation of the circular feature in (001) poles at all temperatures. While floor slip causes reorientation of the grains towards the center on (001) pole figure, roof slip causes reorientation away from the center. At $573 \mathrm{~K}$, the temperature primarily influences the propensity of twinning. $\{130\}\langle 3 \overline{1} 0\rangle$ twin activity is seen to decrease both with strain and as temperature increases, such that it is nearly suppressed at $573 \mathrm{~K}$. Temperature also has an interesting effect on which slip modes are active. While floor slip (001)[100] is dominant from room temperature to $473 \mathrm{~K}$, chimney slip $1 / 2\{110\}\langle 1 \overline{1} 0\rangle$ becomes dominant over floor 
slip (001)[100] at $573 \mathrm{~K}$, at least during the first part of straining. Also, we observe that the contribution of roof slip $1 / 2\{021\}\langle 1 \overline{1} 2\rangle$, decreases from being the second most active at room temperature to the third most active at $573 \mathrm{~K}$. Likewise, in IPC2, as temperature increases from $473 \mathrm{~K}$ to $573 \mathrm{~K}, 1 / 2\{110\}\langle 1 \overline{1} 0\rangle$ slip becomes noticeably dominant and twinning decreases.

Given the good agreement between model and experiment, we analyze the threshold stresses for each slip mode and their own individual dependence on temperature. To elucidate this dependence, we plot in Fig. 5 the variation in the athermal initial slip resistance, $\tau_{0}^{\alpha}$ with temperature predicted by the model. Here we find that the $1 / 2\{110\}\langle 1 \overline{1} 0\rangle$ mode is the most sensitive to temperature while the (010)[100] mode is the least sensitive. These trends are consistent with the experimental test on single crystals reported in [8].

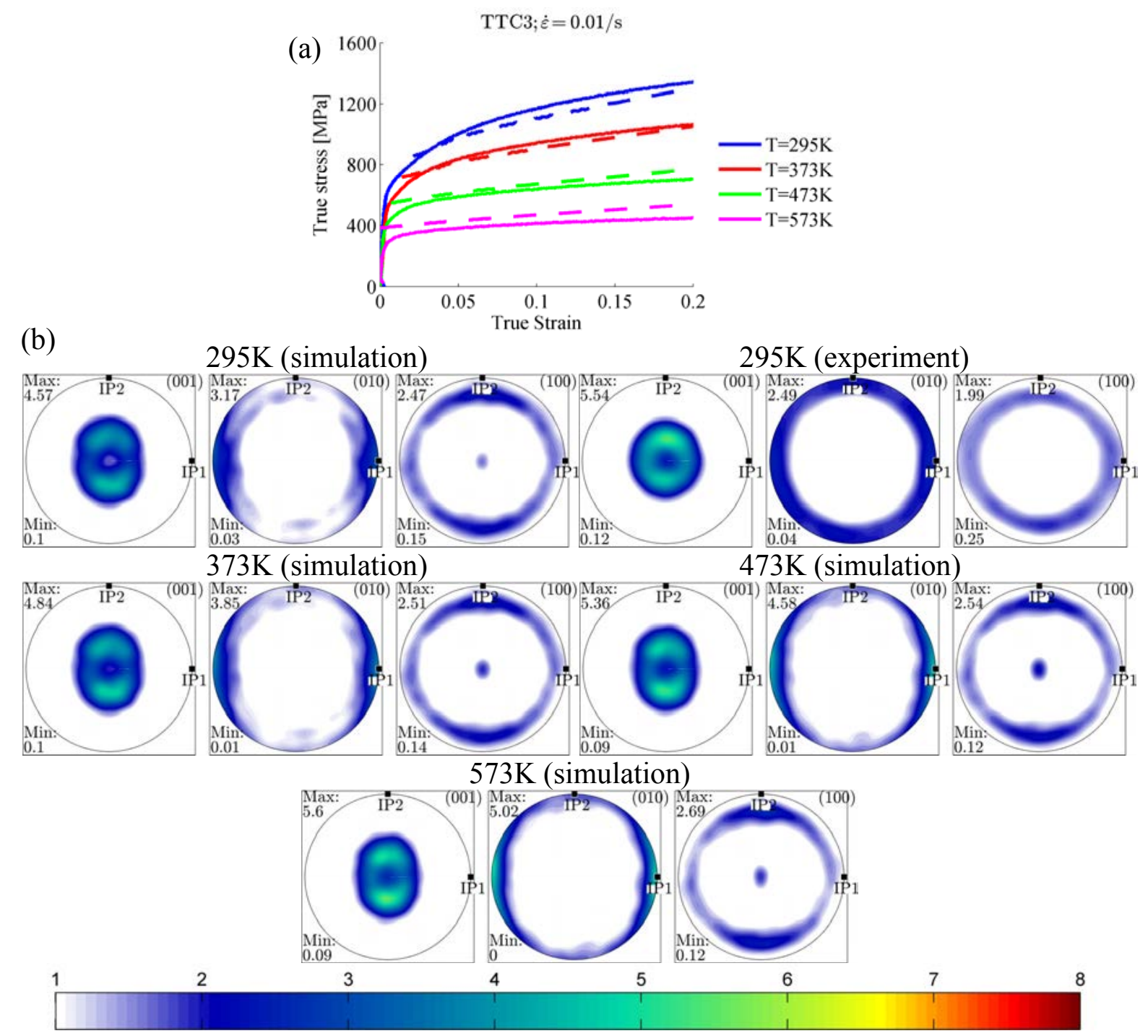


(c)
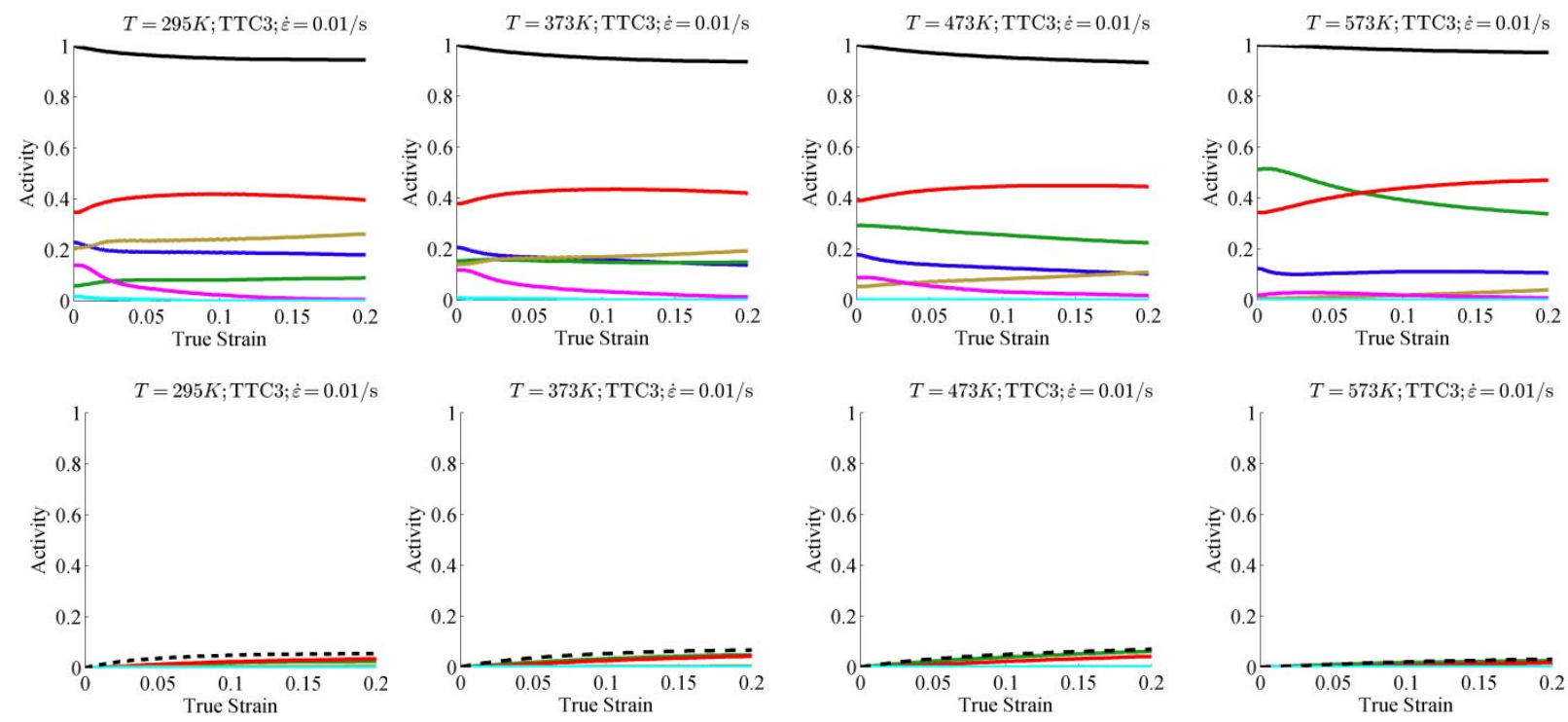

$$
\begin{aligned}
& -(010)[100] \\
& -\{110\}<1-10> \\
& -(001)[100] \\
& -\{021\}<1-12> \\
& \{130\}<3-10> \\
& -\{172\}<3-12> \\
& - \text { Parent material } \\
& -\quad \text { - Twinned material }
\end{aligned}
$$

Fig. 3. Comparison of measured vs. simulated (a) stress-strain response and (b) texture evolution as a function of temperature in the TT directions. (c) Relative activities of active deformation modes. The initial texture was CR.

(b)

(a)
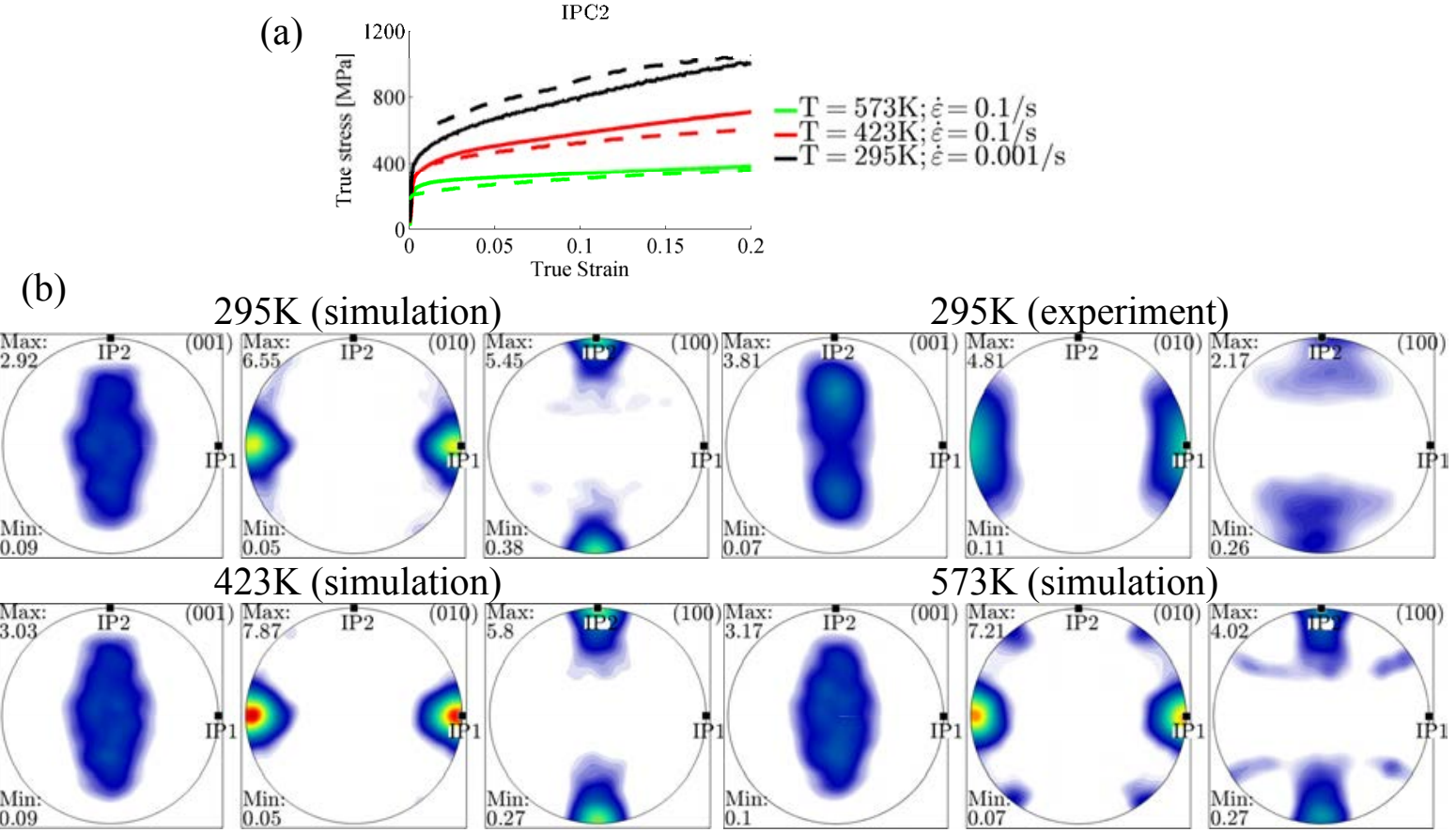


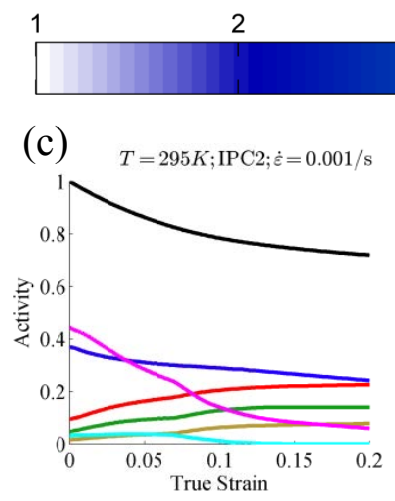

3

4

5

5

$6 \quad 7$ $7 \quad 8$
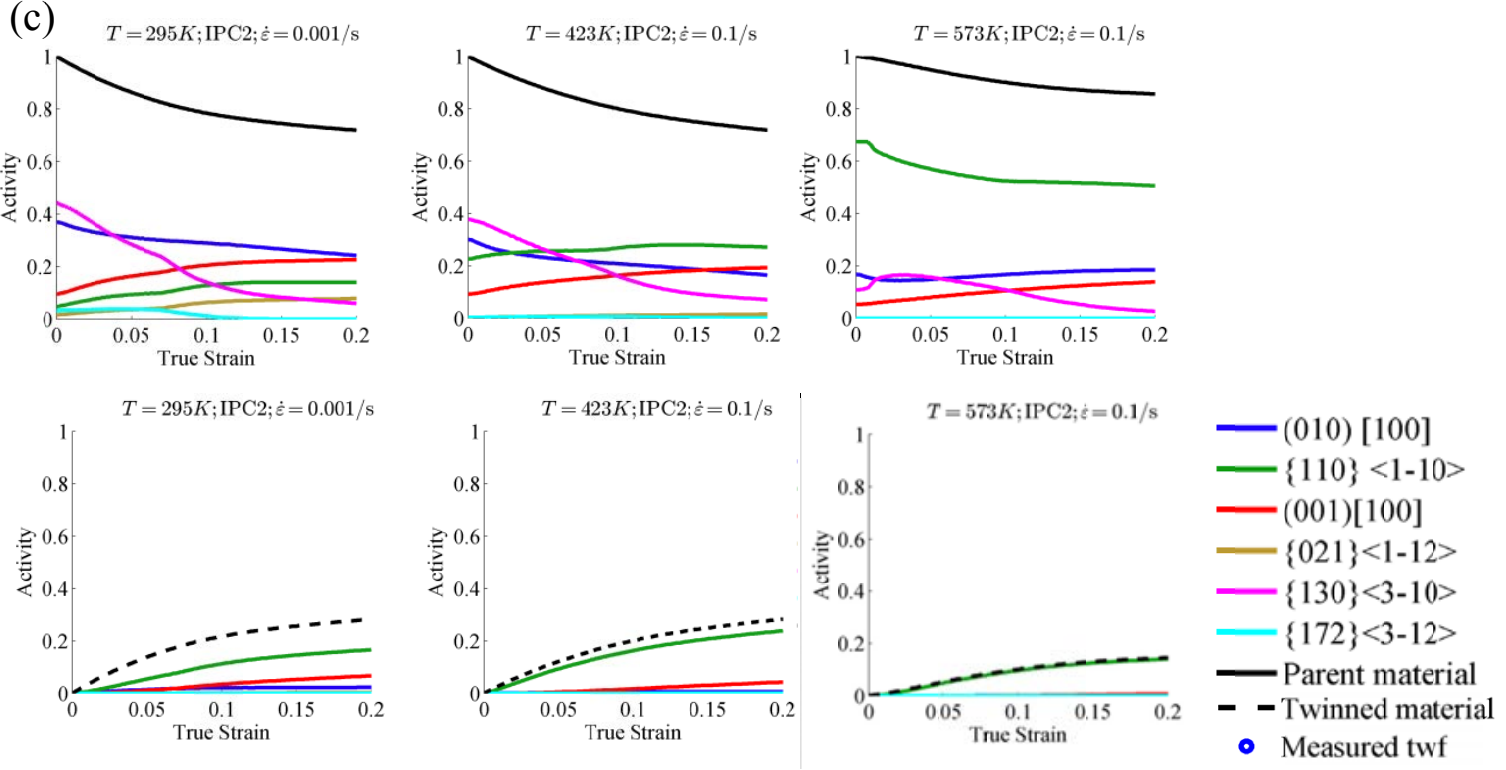

Fig. 4. Comparison of measured vs. simulated (a) stress-strain response and (b) texture evolution as a function of temperature in the IP2 directions. (c) Relative activities of active deformation modes. The initial texture was CR.

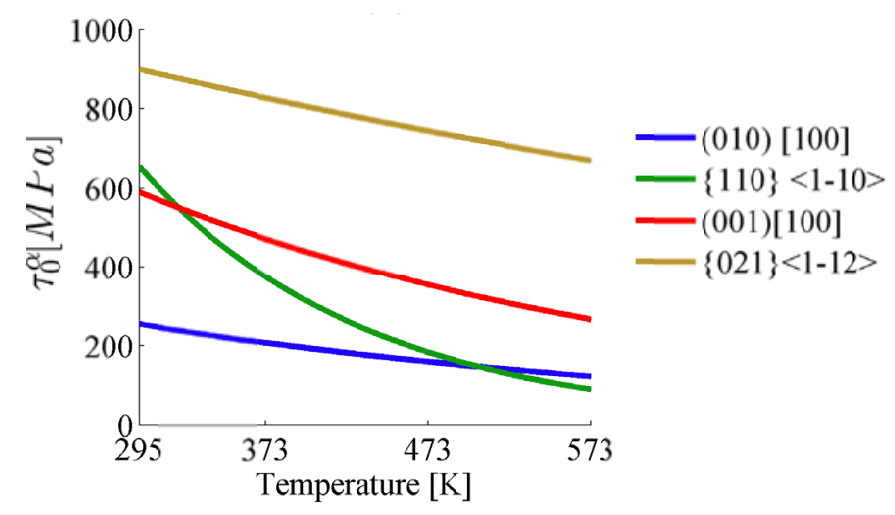

Fig. 5. Predicted dependence of the initial slip resistance on temperature for each slip mode.

\subsection{Texture during plane strain compression and rolling}

To study texture evolution during large strain rolling, the SR material was rolled at $90^{\circ}$ to the original straight rolling direction, i.e., in the IP2 direction, in six rolling passes from $8.89 \mathrm{~mm}$ to $3.56 \mathrm{~mm}$. The total rolling reduction and thickness following each rolling pass were $16 \%$ and $5.96 \mathrm{~mm}, 33 \%$ and $4.98 \mathrm{~mm}, 44 \%$ and $4.27 \mathrm{~mm}, 52 \%$ and $3.56 \mathrm{~mm}$, and $60 \%$ and $3.56 \mathrm{~mm}$, respectively. The rolling was performed at $573 \mathrm{~K}$ with a $203 \mathrm{~mm}$ diameter roll.

In this second portion of the paper, the SR material was rolled at $90^{\circ}$ to the original rolling direction, i.e. in the IP2 direction. Therefore, the TT direction still corresponds to the normal direction (ND), while the IP1 and IP2 directions now correspond to the transverse (TD) and rolling direction (RD), respectively. 
EBSD was used to measure the texture after each rolling pass across the thickness of the sample. From these full through-thickness scans, it was possible to extract spatial textures from the center to the top/bottom surfaces.

Consistent with the experiment, rolling of an $\alpha-U$ sheet to a total reduction of $60 \%$ in five passes at $573 \mathrm{~K}$ was simulated. Two models were used, the VPSC-TW-DD model, which does not allow for heterogeneous boundary conditions and mechanical fields, and later the FE-VPSCTW-DD model, which does.

Figure 6 shows the texture evolution at different rolling reductions from $16 \%$ up to $60 \%$. As shown, the texture changes with straining until they appear to saturate at $44 \%$ rolling reduction.

With VPSC-TW-DD, we performed plane strain compression (PSC) simulations. PSC boundary conditions provided an idealization of the actual rolling process. It best applies to near the center of a rolled sheet and does not account for the alterations in deformation caused by the interactions between the rolls and the sample at the two surfaces of the sheet. The starting microstructure for the model was the SR texture. The deformation temperature and strain rate were set to $573 \mathrm{~K}$ and $0.001 / \mathrm{s}$. The TT direction of the initial SR texture corresponds to the normal direction (ND), while the IP1 and IP2 directions correspond to the transverse (TD) and rolling direction $(\mathrm{RD})$, respectively.

Figure 6a compares the VPSC-TW-DD calculations with the measured textures for the same rolling reductions as measured by EBSD. As seen, the simulation achieves good agreement, considering the complexity of material. Most notable is the development of the two peaks near the TT in the (001) pole figure. The two pronounced peaks lie approximately at $\pm 10-15^{\circ}$ from the IP1-TT plane and exhibit symmetry about the same plane. The small asymmetry, which is unusual for pure PSC deformation, between the intensity peak at the top vs. bottom present in the simulation stems from the asymmetry of the initial texture and the grain co-rotation scheme used in simulations. It is also noted that the two peaks in the simulated textures are slightly closer together as compared to experimental measurements. The model captures the small peak in the (010) pole figure in the IP2 direction, which is now the rolling direction. However, the formation of the peak is slightly faster than is evident from the experimental pole figures. The development of a (100) peak in the IP1 direction, which is now the transverse direction, is captured well while the features close to IP2 exhibit a somewhat different character as compared with the experiment. The last discrepancy that we note lies in the development of a component in the (100) pole figure along the TT direction. The origin of these components will be discussed shortly.

The textures that develop during rolling have some noticeable differences from those that develop in compression (Figs. 2-4). Generally the distribution is an outcome of several activated slip and twinning systems (Fig. 6b), each contributing a different amount to accommodating strain and lattice reorientation. For instance, we see that in TTC of the SR material at 573K, the active slip modes are floor slip (001)[100] and chimney slip $1 / 2\{110\}\langle 1 \overline{1} 0\rangle$; in IPC2 at 573K, they are chimney slip $1 / 2\{110\}\langle 1 \overline{1} 0\rangle$ and wall slip (010)[100]; but in PSC at $573 \mathrm{~K}$, they are floor slip (001)[100], and chimney slip $1 / 2\{110\}\langle 1 \overline{1} 0\rangle$ with slightly different ratio from that in TTC at $573 \mathrm{~K}$. However, we also note that in TTC, $\{130\}\langle 3 \overline{1} 0\rangle$ twinning is not very active but in PSC, it achieves a significant twin volume fraction of $\sim 10 \%$ at a strain of 0.2 and reaches over $20 \%$ with continuous straining (Fig. 6c). Since IPC1 is TD, which is constrained during rolling, there is a compressive stress component acting in this direction that activates $\{130\}\langle 3 \overline{1} 0\rangle$ twinning. By comparing the twin volume fractions after compression during the monotonic compression tests in three directions, we see that IPC1 is most favorable for twinning. In 
addition it can be seen that rolling texture contains features of both TTC (distinct peaks on (001) pole figure) and IPC1 (formation of peaks in IP2 and IP1 directions on (010) and (100) pole figures, respectively) textures. Within the twin domains, the model predicts that chimney slip $1 / 2\{110\}\langle 1 \overline{1} 0\rangle$ and floor slip (001)[100] modes are again most active.

(a)
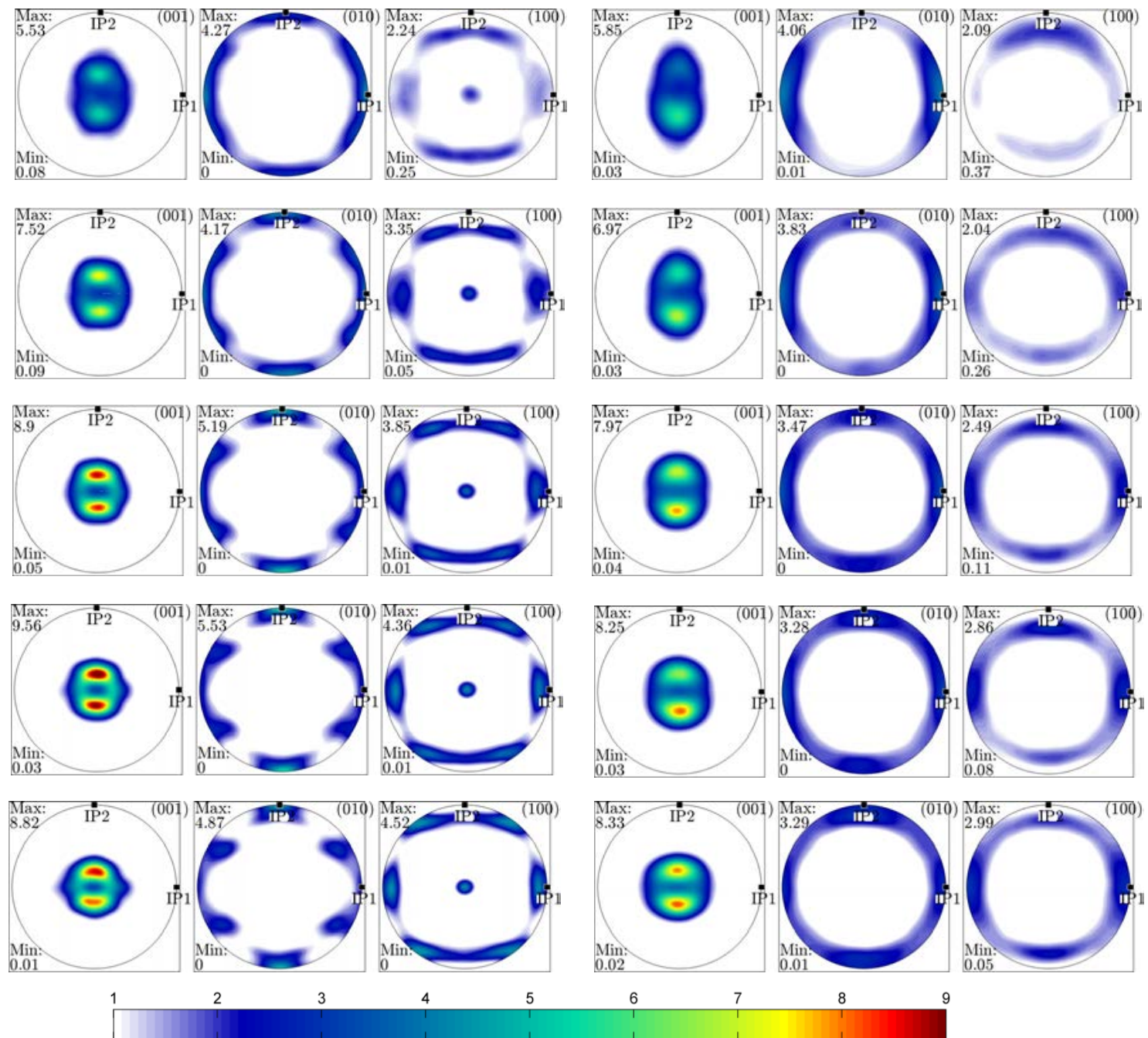

(b)
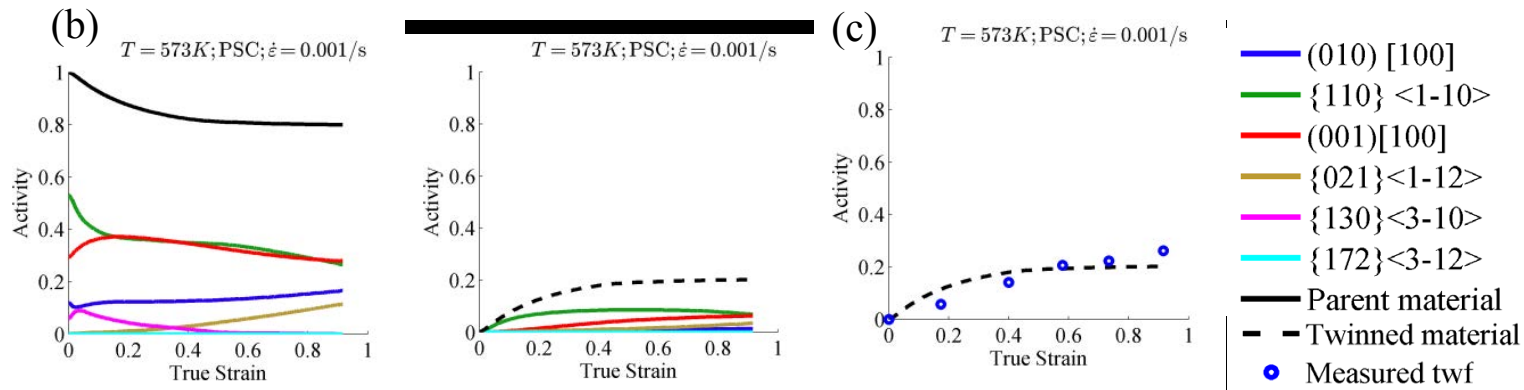

Fig. 6. Plane strain compression of $\alpha-U$ at $573 \mathrm{~K}$ : (a) measured and predicted texture evolution, (b) relative activities of deformation modes, and (c) measured and predicted twin volume 
fraction. The IP1 direction is the transverse direction and IP2 is the rolling direction. The reductions correspond to the following compressive strain along ND: $-0.174,-0.4,-0.58,-0.734$, and -0.916 .

\subsection{Texture gradients}

In this section, we extend our experimental and numerical analysis to examine heterogeneous texture development through the thickness of the sample. The rolling simulations were performed using the FE-VPSC-TW-DD model in the standard ABAQUS software. Not only does this model provide a more representative of the actual rolling process compared to PSC, it also gives us the opportunity to discuss the applicability of the PSC assumption. In the the FEVPSC-TW-DD model, the effects of texture, microstructural evolution, and deformation response are coupled. The mesh consisted of $396 \mathrm{CPE} 4$ bilinear plane strain elements. The initial texture, represented using 1600 individual orientations, was assigned to two elements and spread equally over their eight FE integration points, where each integration point consisted of 200 grains. Based on the geometry of the rolls and the sheet, asymmetry in texture development measured at the surfaces and temperature conditions, the coefficient of friction between roller and $\alpha-\mathrm{U}$ sheet was estimated to be 0.15 . The VPSC calculation for the constitutive response at each integration point was carried out in every time step. For VPSC, the input temperature is $573 \mathrm{~K}$.

As in the experiment, rolling of an $\alpha-\mathrm{U}$ sheet to a total reduction of $60 \%$ in five passes was simulated. FE setup for rolling consisting of the plate and the roller is shown in Fig. 7. The entire five-pass simulation consists of multiple steps. We first lowered the roll and establish contact with the sheet for the first rolling pass. We then imposed a rolling reduction of $16 \%$ by rotating the roll. After the completion of the first rolling pass, the sheet is returned to the origin, the position of the roll is re-adjusted, and the roll is lowered to establish contact with the sheet. Then for the second pass, the roll is rotated to result in a total rolling reduction of $33 \%$. These steps were repeated until a total $60 \%$ rolling reduction is achieved after the fifth pass. State variables, such as dislocation density and crystallographic orientation, were updated and kept throughout the entire simulation.

In the actual rolling process, the deformation state at the sheet surfaces, where the material meets the rolls, can differ from the center. Such gradients in deformation are expected to result in gradients in texture and other microstructure characteristics. The development of texture gradients through the thickness of the rolled sheet has been studied for cubic materials, such as $\mathrm{Cu}$ and $\mathrm{Al}$ and steel [74], with far less complicated behavior than $\alpha$-U. For a material such as $\alpha-$ $\mathrm{U}$, with its complicated constitutive response, deformation gradients generated in rolling could have a more severe effect on the final microstructure of the sheet. Moreover, gradients in texture influence stored energy and grain boundary character distribution; both aspects can influence recrystallization kinetics [75]. 


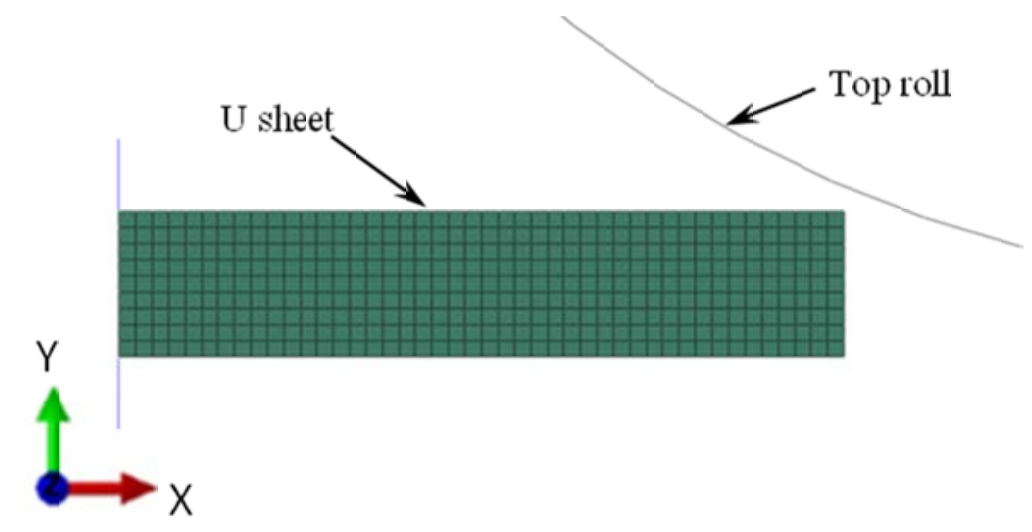

Fig. 7. Simulation set-up for rolling to a total reduction of $60 \%$ in five passes. The initial $2 D$ mesh of 1/2 of the sheet consisting of 396 CPE4 plane strain elements.

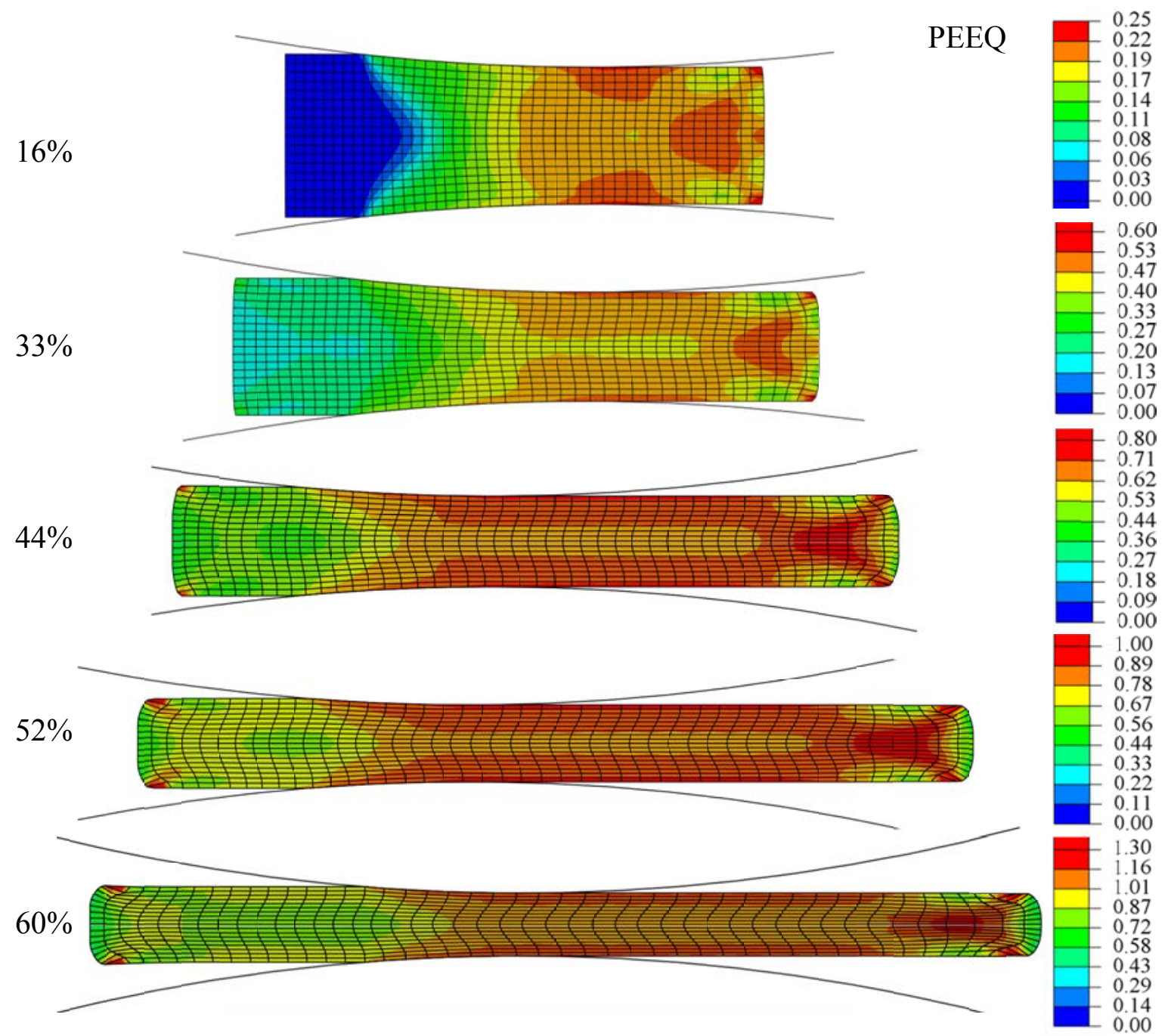

Fig. 8. Fields of equivalent plastic strain (PEEQ) as predicted by the FE-VPSC-TW-DD model. 
Figure 8 shows calculated equivalent plastic strain contours for all five passes. We observe that the FE simulation forecasts that strains develop non-uniformly through the thickness of the sheet, while strain distribution in the rolling direction is largely uniform, except close to the ends. For most of the sheet, apart from the ends, the strains at the surfaces are higher than in the center.

Figure 9 compares the measured and predicted textures in this representative surface region. Notably, the FE-VPSC textures agree well with experiment. As expected, these textures are also different from those away from the surface towards the center. The texture evolution across the entire thickness was measured by EBSD and away from the surface across the thickness was predicted by stand-alone VPSC-TW-DD under PSC as shown in Fig. 6. The FE model predicts a similar symmetric texture for this area. Comparing the bulk EBSD and FE-center textures with the predicted FE-texture at the surface, we find that the texture in the center region of the sheet, but not the surface textures, can be predicted by the plane strain compression model.

To determine the severity in the variation, local textures were extracted at the top and bottom locations from the full through-the-thickness EBSD scans. Figure 9 shows the texture at the bottom surface. We see that the texture that develops at the bottom surface is developing asymmetry in the two predominant peaks in (001) pole figure. While the texture at bottom develops the lower peak to be stronger, the texture at the top surface developed the upper peak to be stronger. The shear stress state imposed by the rolls at the surface causes the asymmetry in the intensities of these peaks. For the texture close to the bottom surface, the higher activity of floor slip in the grains with orientations corresponding to the lower portion of the (001) pole figure forms the stronger bottom peak. Additionally, a rigid body rotation enhances the asymmetry because it develops away from the center and towards the surface of the sheet.

In the above, we have demonstrated that the multi-scale FE-VPSC-TW-DD model can capture the development of texture gradients in $\alpha-\mathrm{U}$, a metal with a highly anisotropic plastic response, despite the previously indicated discrepancies between the predictions and experiments for PSC. The ability to predict these gradients is particularly important for understanding recrystallization during post-rolling heat treatments. Gradients in texture will influence many microstructural features believed to affect recrystallization, such as crystallographic orientation, gradients in orientation, gradients in stored energy in grains and near grain boundaries, and the distribution in the properties of grain boundaries. In this way, texture can impact the percentage of recrystallized material and gradients in recrystallization grain sizes and texture. So, use of this numerical tool, in spite of the computational expense, can lead to better understanding and predictions of recrystallization kinetics and finally recrystallized microstructure. 


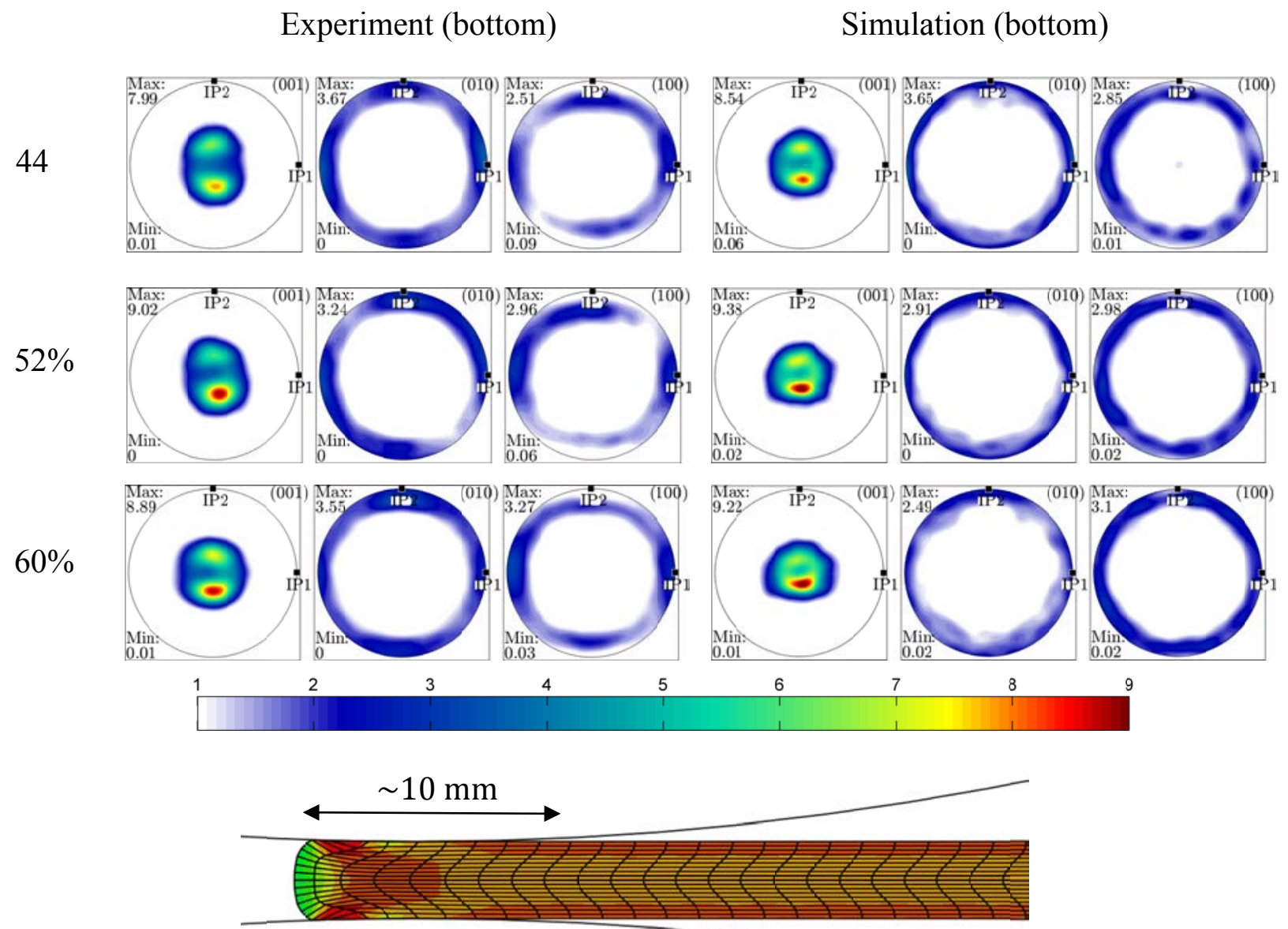

Fig. 9. Pole figures showing predicted texture at a polycrystalline material point near the surface of the sheet. Texture was measured approximately $10 \mathrm{~mm}$ from the exit, sufficiently far to avoid any non-uniform strain distribution characteristic for the ends. The IP1 direction is the transverse direction and IP2 is the rolling direction. The reductions correspond to the following compressive strain along ND: $-0.58,-0.734$, and -0.916 .

\section{Conclusions}

In this work, we studied the mechanical response and microstructure evolution of $\alpha$-U during simple compression and large strain rolling. We carried out a comprehensive set of mechanical tests over a wide range of temperatures and characterized the microstructure using EBSD as a function of deformation mode and strain level. At the same time, we used a dislocation-density based, multi-scale constitutive model within a self-consistent homogenization in order to determine the dominant deformation mechanisms underlying texture evolution. We show that with a single set of material parameters, we achieve good agreement with measurements of texture and stress-strain response. From the analysis, we find that in both through-thickness compression (TTC) at $573 \mathrm{~K}$ and rolling at $573 \mathrm{~K}$, the active slip modes are floor slip (001)[100] and chimney slip $1 / 2\{110\}\langle 1 \overline{1} 0\rangle$ with slightly different ratios. However, while $\{130\}\langle 3 \overline{1} 0\rangle$ twinning is not active in TTC compression, we observe significant deformation twinning during rolling. This twin mode does little to accommodate the reduction in the plate normal direction, 
but does markedly contribute to the extension in the rolling direction. We show that in-plane, room temperature deformation is also dominated by twinning, which decreases with increasing temperature. In the case of rolling, we predict that floor slip (001)[100] results in the formation of two pronounced (001) texture peaks $10-15^{\circ}$ away from the normal toward the rolling direction. Finally, we demonstrate that the multi-scale FE-VPSC model can capture the development of texture gradients in $\alpha-U$, a metal with a highly anisotropic plastic response. The ability to predict these gradients is particularly important for understanding recrystallization during post-rolling heat treatments.

\section{Acknowledgements}

M. Z. acknowledges support from the CEPS Graduate Fellowships program at the University of New Hampshire (UNH). M. K. acknowledges subcontract, NO. 277871, granted by Los Alamos National Laboratory to UNH. I. J. B. and R. J. M. acknowledge support by a Laboratory Directed Research and Development grant 20140630ER.

\section{References}

[1] R.W. Cahn, Plastic deformation of alpha-uranium; twinning and slip, Acta Metallurgica, 1 (1953) 49-70.

[2] R.J. McCabe, L. Capolungo, P.E. Marshall, C.M. Cady, C.N. Tomé, Deformation of wrought uranium: Experiments and modeling, Acta Materialia, 58 (2010) 5447-5459.

[3] M. Ardeljan, R.J. McCabe, I.J. Beyerlein, M. Knezevic, Explicit incorporation of deformation twins into crystal plasticity finite element models, Computer Methods in Applied Mechanics and Engineering, 295 (2015) 396-413.

[4] P.G. Partridge, The crystallography and deformation modes of hexagonal close-packed metals, Metallurgia Revised, 12 (1967) 169.

[5] M.H. Yoo, Slip, twinning, and fracture in hexagonal close-packed metals, Metall. Mater. Trans. A, 12 (1981) 409-418.

[6] M. Lentz, M. Klaus, M. Wagner, C. Fahrenson, I.J. Beyerlein, M. Zecevic, W. Reimers, M. Knezevic, Effect of age hardening on the deformation behavior of an Mg-Y-Nd alloy: In-situ Xray diffraction and crystal plasticity modeling, Materials Science and Engineering: A, 628 (2015) 396-409.

[7] M.H. Yoo, Slip modes of alpha uranium, Journal of Nuclear Materials, 26 (1968) 307-318.

[8] J.S. Daniel, B. Lesage, P. Lacombe, The influence of temperature on slip and twinning in uranium, Acta Metallurgica, 19 (1971) 163-173.

[9] R.W. Cahn, Twinning and slip in $\alpha$-uranium, Acta Crystallographica, 4 (1951) 470.

[10] R.G. Anderson, J.W. Bishop, The effect of neutron irradiation and thermal cycling on permanent deformations in uranium under load, in: Symposium on uranium and graphite, The Institute of Metals, London 1962, pp. 17-23.

[11] E.S. Fisher, H.J. McSkimin, Adiabatic elastic moduli of single crystal alpha Uranium, Journal of Applied Physics, 29 (1958) 1473 - 1484.

[12] R.A. Lebensohn, C.N. Tomé, A self-consistent viscoplastic model: prediction of rolling textures of anisotropic polycrystals, Materials Science and Engineering: A, 175 (1994) 71-82. [13] A.D. Rollett, Comparison of experimental and theoretical texture development in alphauranium, in: T.C. Lowe, A.D. Rollett, P.S. Follansbee, G.S. Daehn (Eds.) Symposium on Modeling the Deformation of Crystalline Solids, TMS, Warrendale, PA, 1991, pp. 361-368. 
[14] D.W. Brown, M.A.M. Bourke, B. Clausen, D.R. Korzekwa, R.C. Korzekwa, R.J. McCabe, T.A. Sisneros, D.F. Teter, Temperature and direction dependence of internal strain and texture evolution during deformation of uranium, Materials Science and Engineering: A, 512 (2009) 6775.

[15] C.S. Choi, M. Staker, Neutron diffraction texture study of deformed uranium plates, J Mater Sci, 31 (1996) 3397-3402.

[16] M. Knezevic, L. Capolungo, C.N. Tomé, R.A. Lebensohn, D.J. Alexander, B. Mihaila, R.J. McCabe, Anisotropic stress-strain response and microstructure evolution of textured $\alpha$-uranium, Acta Materialia, 60 (2012) 702-715.

[17] M. Knezevic, J. Crapps, I.J. Beyerlein, D.R. Coughlin, K.D. Clarke, R.J. McCabe, Anisotropic modeling of structural components using embedded crystal plasticity constructive laws within finite elements, International Journal of Mechanical Sciences, 105 (2016) 227-238. [18] M. Knezevic, R.J. McCabe, C.N. Tomé, R.A. Lebensohn, S.R. Chen, C.M. Cady, G.T. Gray Iii, B. Mihaila, Modeling mechanical response and texture evolution of $\alpha$-uranium as a function of strain rate and temperature using polycrystal plasticity, International Journal of Plasticity, 43 (2013) 70-84.

[19] C.A. Calhoun, E. Garlea, R.P. Mulay, T.A. Sisneros, S.R. Agnew, Investigation of the effect of thermal residual stresses on deformation of $\alpha$-uranium through neutron diffraction measurements and crystal plasticity modeling, Acta Materialia, 85 (2015) 168-179. [20] U.F. Kocks, C.N. Tome', H.-R. Wenk, Texture and Anisotropy, Cambridge University Press, Cambridge, 1998.

[21] M. Knezevic, B. Drach, M. Ardeljan, I.J. Beyerlein, Three dimensional predictions of grain scale plasticity and grain boundaries using crystal plasticity finite element models, Computer Methods in Applied Mechanics and Engineering, 277 (2014) 239-259.

[22] M. Knezevic, D.J. Savage, A high-performance computational framework for fast crystal plasticity simulations, Computational Materials Science, 83 (2014) 101-106.

[23] M. Knezevic, H.F. Al-Harbi, S.R. Kalidindi, Crystal plasticity simulations using discrete Fourier transforms, Acta Materialia, 57 (2009) 1777-1784.

[24] M. Knezevic, S.R. Kalidindi, D. Fullwood, Computationally efficient database and spectral interpolation for fully plastic Taylor-type crystal plasticity calculations of face-centered cubic polycrystals, International Journal of Plasticity, 24 (2008) 1264-1276.

[25] M. Knezevic, S.R. Kalidindi, R.K. Mishra, Delineation of first-order closures for plastic properties requiring explicit consideration of strain hardening and crystallographic texture evolution, International Journal of Plasticity, 24 (2008) 327-342.

[26] M. Knezevic, N.W. Landry, Procedures for reducing large datasets of crystal orientations using generalized spherical harmonics, Mechanics of Materials, 88 (2015) 73-86.

[27] D.J. Savage, M. Knezevic, Computer implementations of iterative and non-iterative crystal plasticity solvers on high performance graphics hardware, Computational Mechanics 56 (2015) 677-690.

[28] T. Fast, M. Knezevic, S.R. Kalidindi, Application of microstructure sensitive design to structural components produced from hexagonal polycrystalline metals, Computational Materials Science, 43 (2008) 374-383.

[29] M. Knezevic, S.R. Kalidindi, Fast computation of first-order elastic-plastic closures for polycrystalline cubic-orthorhombic microstructures, Computational Materials Science, 39 (2007) 643-648. 
[30] H.F. Al-Harbi, M. Knezevic, S.R. Kalidindi, Spectral approaches for the fast computation of yield surfaces and first-order plastic property closures for polycrystalline materials with cubictriclinic textures, CMC: Computers, Materials, \& Continua, 15 (2010) 153-172.

[31] M. Knezevic, M. Zecevic, I.J. Beyerlein, A. Bhattacharyya, R.J. McCabe, Predicting Texture Evolution in Ta and Ta-10W Alloys Using Polycrystal Plasticity, JOM, 67 (2015) 26702674.

[32] B.S. Fromm, B.L. Adams, S. Ahmadi, M. Knezevic, Grain size and orientation distributions: Application to yielding of $\alpha$-titanium, Acta Materialia, 57 (2009) 2339-2348. [33] S.R. Kalidindi, H.K. Duvvuru, M. Knezevic, Spectral calibration of crystal plasticity models, Acta Materialia, 54 (2006) 1795-1804.

[34] S.R. Kalidindi, M. Knezevic, S. Niezgoda, J. Shaffer, Representation of the orientation distribution function and computation of first-order elastic properties closures using discrete Fourier transforms, Acta Materialia, 57 (2009) 3916-3923.

[35] B. Mihaila, M. Knezevic, A. Cardenas, Three orders of magnitude improved efficiency with high - performance spectral crystal plasticity on GPU platforms, International Journal for Numerical Methods in Engineering, 97 (2014) 785-798.

[36] J.B. Shaffer, M. Knezevic, S.R. Kalidindi, Building texture evolution networks for deformation processing of polycrystalline fcc metals using spectral approaches: Applications to process design for targeted performance, International Journal of Plasticity, 26 (2010) 11831194.

[37] M. Jahedi, M.H. Paydar, S. Zheng, I.J. Beyerlein, M. Knezevic, Texture evolution and enhanced grain refinement under high-pressure-double-torsion, Materials Science and Engineering: A, 611 (2014) 29-36.

[38] R.A. Lebensohn, C.N. Tomé, A self-consistent anisotropic approach for the simulation of plastic deformation and texture development of polycrystals: Application to zirconium alloys, Acta Metallurgica et Materialia, 41 (1993) 2611-2624.

[39] J.W. Hutchinson, Bounds and self-consistent estimates for creep of polycrystalline materials, Proceedings of the Royal Society of London. Series A, Mathematical and Physical Sciences, (1976) 101-126.

[40] R.J. Asaro, A. Needleman, Texture development and strain hardening in rate dependent polycrystals, Acta Metallurgica et Materialia, 33 (1985) 923-953.

[41] M. Knezevic, I.J. Beyerlein, M.L. Lovato, C.N. Tomé, A.W. Richards, R.J. McCabe, A strain-rate and temperature dependent constitutive model for BCC metals incorporating nonSchmid effects: Application to tantalum-tungsten alloys, International Journal of Plasticity, 62 (2014) 93-104.

[42] M. Knezevic, J.S. Carpenter, M.L. Lovato, R.J. McCabe, Deformation behavior of the cobalt-based superalloy Haynes 25: Experimental characterization and crystal plasticity modeling, Acta Materialia, 63 (2014) 162-168.

[43] G. Proust, C.N. Tomé, G.C. Kaschner, Modeling texture, twinning and hardening evolution during deformation of hexagonal materials, Acta Materialia, 55 (2007) 2137-2148.

[44] M. Zecevic, M. Knezevic, I.J. Beyerlein, C.N. Tomé, An elasto-plastic self-consistent model with hardening based on dislocation density, twinning and de-twinning: Application to strain path changes in HCP metals, Materials Science and Engineering: A, 638 (2015) 262-274. [45] M. Knezevic, M. Zecevic, I.J. Beyerlein, J.F. Bingert, R.J. McCabe, Strain rate and temperature effects on the selection of primary and secondary slip and twinning systems in HCP Zr, Acta Materialia, 88 (2015) 55-73. 
[46] I.J. Beyerlein, C.N. Tomé, A dislocation-based constitutive law for pure Zr including temperature effects, International Journal of Plasticity, 24 (2008) 867-895.

[47] M. Knezevic, I.J. Beyerlein, D.W. Brown, T.A. Sisneros, C.N. Tomé, A polycrystal plasticity model for predicting mechanical response and texture evolution during strain-path changes: Application to beryllium, International Journal of Plasticity, 49 (2013) 185-198. [48] M. Ardeljan, M. Knezevic, T. Nizolek, I.J. Beyerlein, N.A. Mara, T.M. Pollock, A study of microstructure-driven strain localizations in two-phase polycrystalline $\mathrm{HCP} / \mathrm{BCC}$ composites using a multi-scale model, International Journal of Plasticity, 74 (2015) 35-57.

[49] M. Ardeljan, I.J. Beyerlein, M. Knezevic, A dislocation density based crystal plasticity finite element model: Application to a two-phase polycrystalline $\mathrm{HCP} / \mathrm{BCC}$ composites, Journal of the Mechanics and Physics of Solids, 66 (2014) 16-31.

[50] M. Lentz, M. Klaus, I.J. Beyerlein, M. Zecevic, W. Reimers, M. Knezevic, In situ X-ray diffraction and crystal plasticity modeling of the deformation behavior of extruded $\mathrm{Mg}-\mathrm{Li}-(\mathrm{Al})$ alloys: An uncommon tension-compression asymmetry, Acta Materialia, 86 (2015) 254-268. [51] M. Knezevic, I.J. Beyerlein, T. Nizolek, N.A. Mara, T.M. Pollock, Anomalous Basal Slip Activity in Zirconium under High-strain Deformation, Materials Research Letters, 1 (2013) 133140.

[52] J.S. Carpenter, T. Nizolek, R.J. McCabe, M. Knezevic, S.J. Zheng, B.P. Eftink, J.E. Scott, S.C. Vogel, T.M. Pollock, N.A. Mara, I.J. Beyerlein, Bulk texture evolution of nanolamellar Zr$\mathrm{Nb}$ composites processed via accumulative roll bonding, Acta Materialia, 92 (2015) 97-108. [53] M. Zecevic, M. Knezevic, A dislocation density based elasto-plastic self-consistent model for the prediction of cyclic deformation: Application to Al6022-T4, International Journal of Plasticity, 72 (2015) 200-217.

[54] M. Jahedi, M. Ardeljan, I.J. Beyerlein, M.H. Paydar, M. Knezevic, Enhancement of orientation gradients during simple shear deformation by application of simple compression, Journal of Applied Physics, 117 (2015) 214309.

[55] J. Segurado, R.A. Lebensohn, J. Llorca, C.N. Tomé, Multiscale modeling of plasticity based on embedding the viscoplastic self-consistent formulation in implicit finite elements, International Journal of Plasticity, 28 (2012) 124-140.

[56] M. Knezevic, R.A. Lebensohn, O. Cazacu, B. Revil-Baudard, G. Proust, S.C. Vogel, M.E. Nixon, Modeling bending of $\alpha$-titanium with embedded polycrystal plasticity in implicit finite elements, Materials Science and Engineering: A, 564 (2013) 116-126.

[57] M. Knezevic, M. Jahedi, Y.P. Korkolis, I.J. Beyerlein, Material-based design of the extrusion of bimetallic tubes, Computational Materials Science, 95 (2014) 63-73.

[58] M. Knezevic, R.J. McCabe, R.A. Lebensohn, C.N. Tomé, B. Mihaila, Finite element implementation of a self-consistent polycrystal plasticity model: Application to $\alpha$-uranium, Proceedings: Volume 2: Materials Properties, Characterization, and Modeling TMS (The Minerals, Metals \& Materials Society), in, 2012, pp. 789-796.

[59] R. Hill, Generalized constitutive relations for incremental deformation of metal crystals by multislip, Journal of the Mechanics and Physics of Solids, 14 (1966) 95-102.

[60] M. Zecevic, R.J. McCabe, M. Knezevic, Spectral database solutions to elasto-viscoplasticity within finite elements: Application to a cobalt-based FCC superalloy, International Journal of Plasticity, 70 (2015) 151-165.

[61] T.J.R. Hughes, J. Winget, Finite rotation effects in numerical integration of rate constitutive equations arising in large-deformation analysis, International Journal for Numerical Methods in Engineering, 15 (1980) 1862-1867. 
[62] M. Zecevic, R.J. McCabe, M. Knezevic, A new implementation of the spectral crystal plasticity framework in implicit finite elements, Mechanics of Materials, 84 (2015) 114-126.

[63] E.S. Fisher, Temperature dependence of the elastic moduli in alpha uranium single crystals, part IV (298 to $923 \mathrm{~K}$ ), Journal of Nuclear Materials, 18 (1966) 39-54.

[64] M. Knezevic, R.J. McCabe, R.A. Lebensohn, C.N. Tomé, C. Liu, M.L. Lovato, B. Mihaila, Integration of self-consistent polycrystal plasticity with dislocation density based hardening laws within an implicit finite element framework: Application to low-symmetry metals, Journal of the Mechanics and Physics of Solids, 61 (2013) 2034-2046.

[65] I.J. Beyerlein, R.J. McCabe, C.N. Tome, Stochastic processes of $\{1012\}$ deformation twinning in hexagonal close-packed polycrystalline zirconium and magnesium, International Journal for Multiscale Computational Engineering, 9 (2011) 459-480.

[66] I.J. Beyerlein, R.J. McCabe, C.N. Tomé, Effect of microstructure on the nucleation of deformation twins in polycrystalline high-purity magnesium: A multi-scale modeling study, Journal of the Mechanics and Physics of Solids, 59 (2011) 988-1003.

[67] M. Knezevic, T. Nizolek, M. Ardeljan, I.J. Beyerlein, N.A. Mara, T.M. Pollock, Texture evolution in two-phase $\mathrm{Zr} / \mathrm{Nb}$ lamellar composites during accumulative roll bonding, International Journal of Plasticity, 57 (2014) 16-28.

[68] R. Madec, B. Devincre, L. Kubin, T. Hoc, D. Rodney, The role of collinear interaction in dislocation-induced hardening, Science, 301 (2003) 1879-1882.

[69] H. Mecking, U.F. Kocks, Kinetics of flow and strain-hardening., Acta Metallurgica et Materialia, 29 (1981) 1865-1875.

[70] R. Madec, B. Devincre, L.P. Kubin, From Dislocation Junctions to Forest Hardening, Physical Review Letters, 89 (2002) 255508.

[71] U. Essmann, H. Mughrabi, Annihilation of dislocations during tensile and cyclic deformation and limits of dislocation densities, Philosophical Magazine A, 40 (1979) 731-756. [72] C. Tomé, C. Necker, R. Lebensohn, Mechanical anisotropy and grain interaction in recrystallized aluminum, Metallurgical and Materials Transactions A, 33 (2002) 2635-2648. [73] I.J. Beyerlein, J.S. Carpenter, A. Hunter, L.S. Tóth, W. Skrotzki, Nano-enabled orientation alignment via extreme shear strains, Scripta Materialia, 98 (2015) 52-55.

[74] O. Engler, C. Tomé, M.-Y. Huh, A study of through-thickness texture gradients in rolled sheets, Metallurgical and Materials Transactions A, 31 (2000) 2299-2315.

[75] R.J. McCabe, A.W. Richards, D. Coughlin, K.D. Clarke, I.J. Beyerlein, M. Knezevic, Microstructure effects on the recrystallization of low-symmetry alpha-uranium, Journal of Nuclear Materials, (2015). 